\title{
A Study of the Viscous Effects over an Acoustic Liner using the Linearised Navier-Stokes equations in the Frequency Domain
}

BORJA PASCUAL JOSÉ 



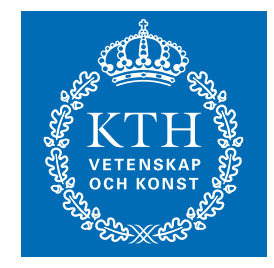

\title{
A study of the viscous effects over an acoustic liner using the Linearised Navier-Stokes equations in the frequency domain
}

\author{
BORJA PASCUAL JOSÉ \\ Master's Thesis at the Department of Aeronautical and Vehicle Engineering \\ Supervisor: Ciarán O'Reilly \\ Examiner: Gunilla Efraimsson
}





\begin{abstract}
New noise regulations for civil aviation restrict the sound level that engines can emit to a great extent. Acoustic liners are the most widespread solution in order to damp sound in aircraft engines. Usually the Linearised Euler equations (LEE) are used to calculate the sound propagation through the engine. In addition, the viscous effects close to the near wall region, where the acoustic liner is located, are considered by the Myers impedance boundary condition. However, this boundary condition has been proved to be ill-posed and to not fully capture the viscous acoustics. Hence, a different approach is taken by using the full Linearised Navier-Stokes equations. In order to assess the validity of this method a computational model is created to reproduce the experimental work done by Aurégan et.al. where the scattering matrix of a two source model is measured and time domain simulations are done using LEE and Myers boundary condition in order to compare them. An improvement, with respect to the inviscid time domain simulations, is achieved when the upstream educed impedance values are used. Therefore, the Myers impedance boundary condition can still be used in numerical impedance eduction codes and the obtained values render good results if a viscous solution is adopted. Also, considering a viscous solution implies that both the hydrodynamic and the acoustic boundary layer need to be resolved. Nonetheless, the latter is very small compared to the hydrodynamic one and its inclusion will result in a very fine mesh that might increase the computational time. Thus, a second study is done in order to assess the importance of the acoustic boundary layer in these calculations, and to determine if some assumption can be applied in order to reduce the computational effort. To that purpose, all the simulations are done in the frequency domain since it is a lighter computational method than the time domain. Results of this second test case show that the resolution of the acoustic boundary layer is not a crucial factor in order to achieve an accurate solution.
\end{abstract}




\section{Acknowledgements}

I would like to thank my supervisor Ciarán O'Reilly for giving me the opportunity to work with a project related to my interests. I also appreciate his help and guidance as well as his valuable comments. I am immensely grateful for the opportunity of doing my thesis within a research group at KTH, and I would like to express my deepest gratitude to my examiner, Gunilla Efraimsson, and all the members of the group for making me feel always part of it. I would also like to thank PhD students, Stefan Sack and Wei Na, for their time and valuable discussions and inputs with COMSOL.

Finally, I would like to express my gratitude to my parents and family for supporting me always and, specially, during these two years away from home. Thanks a lot for always being there for me.

Borja Pascual José. 


\section{Contents}

Contents $\quad$ v

1 Introduction 1

1.1 Background . . . . . . . . . . . . . . . . . . 2

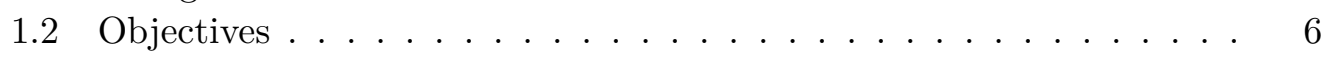

2 Fundamentals of fluid dynamics $\quad 9$

2.1 Governing Equations . . . . . . . . . . . . . . . . . . . . . 9

2.2 Boundary Layer . . . . . . . . . . . . . . . . . . . . . . . . 10

2.3 Turbulence . . . . . . . . . . . . . . . . . 11

2.3.1 Reynolds Average Navier Stokes Equations . . . . . . . . . 11

2.3.2 Turbulence models . . . . . . . . . . . . . . . . . . . . 12

3 Sound propagation in ducts $\quad 13$

3.1 Fundamentals of linear acoustics . . . . . . . . . . . . . . . 13

3.1.1 Scattering matrix and plane wave decomposition . . . . . . 15

3.2 Impedance eduction methods . . . . . . . . . . . . . . . . . 16

3.3 Computational methods for sound propagation in a duct with uniform mean flow . . . . . . . . . . . . . . 17

4 Methodology 19

4.1 Test Case 1: No mean flow . . . . . . . . . . . . . . . . . 19

4.2 Test Case 2: Uniform mean flow . . . . . . . . . . . . . . . 21

4.2 .1 Stage I: Background Flow . . . . . . . . . . . . . . . . 22

4.2 .2 Stage II: Acoustic Propagation . . . . . . . . . . . . . . . 23

4.3 Study Case 1: Viscous effects in a local reacting wall . . . . . . . . . 24

4.4 Study Case 2: Acoustic boundary layer sensitivity study . . . . . . . 26

5 Results and Validation $\quad 27$

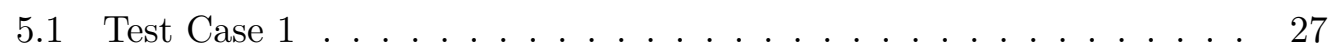

5.2 Test Case $2 \ldots \ldots \ldots \ldots \ldots$

5.2 .1 Impedance sensitivity study . . . . . . . . . . . . . 33

5.3 Study Case $1 \ldots \ldots \ldots$. . . . . . . . . . . . . . . . . . 38

5.4 Study Case $2 \ldots \ldots \ldots \ldots$ 
6 Discussion $\quad 45$

$\begin{array}{lll}7 & \text { Conclusions } & 49\end{array}$

A Data for Test Case 2 with $M=0.079$

B Data for Test Case 2 with $M=0.172$

C Data for Test Case 2 with $M=0.255$

$\begin{array}{lc}\text { Bibliography } & 63\end{array}$ 


\section{Chapter 1}

\section{Introduction}

Civil aviation has increased notably over the past years and predictions indicate the number of aircraft will continue raising [18]. It is obvious that the growth in the number of flights will result in an increment of environmental noise. According to the World Health Organization [8] noise related issues, such as annoyance or sleep disturbance, can cause several short- and long-term health problems. European Union policies also take these problems into consideration by establishing a normative that regulates air traffic around airports. These regulations are in constant revision and their impact on airports economics has been recently studied [27]. However, not everyone reacts the same way to noise disturbances which makes it complicated to establish a clear cause-effect relationship, thus, adding a great amount of uncertainties to the valuation process. Either way, a reduction in noise level would allow airports to operate a larger number of flights per day which could increase its profitability. To this purpose, a lot of research effort has been done in order to find novel methods, or to improve existing methodologies, that enhance the design of more efficient sound damping systems.

In particular, aircraft have several noise sources but engines are the most important contribution. Acoustic liners are a widely used solution to damp sound in aero engines. Usually acoustic liners, shown in Fig 1.1, are installed in the nacelle and consist of a perforated sheet with cavities underneath that are responsible for the dissipation process, and are designed to work more efficiently in a specific frequency range. The vortex shedding generated around the cavities transforms the acoustic energy into rotational kinetic energy, which is in the end dissipated by means of molecular viscosity [29].

Hence, in this chapter an overview of acoustic liners current state of the art for aero engines applications is presented. In addition, a discussion on the scope of this thesis is done in order to clearly stablish its goals and delimitations. 


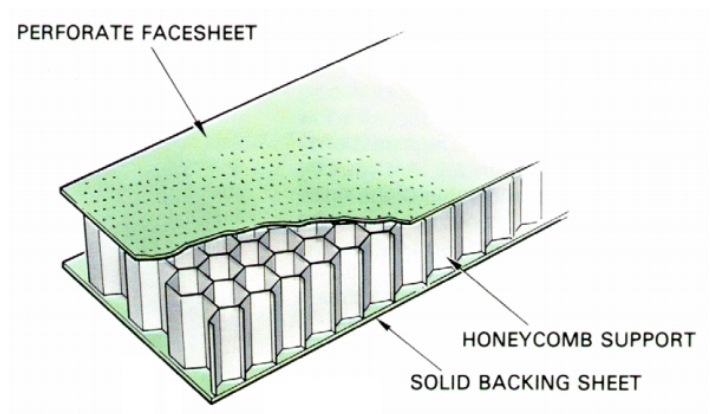

Figure 1.1: Schematic of a single layer perforated acoustic liner [7]

\subsection{Background}

Current noise regulations require engines to obtain a certification based on the Effective Perceived Noise Level (EPNL). For manufacturers, the best way to achieve this data is to perform tests. However, this is a very costly procedure and it does not allow for flexibility since a prototype is needed, thus, making difficult the addition of further modifications. Hence, a more cost-effective option is the use of Computational Aero-Acoustics (CAA). This is a more economic solution and it facilitates the introduction of modifications, thus, being a good tool for optimization processes.

In Fig 1.2 the different numerical approaches found in the literature are summarized. Despite the impedance is mainly a frequency domain boundary condition, time domain solutions seem to prevail. It is worth noting that the main advantage of a time domain approach is the facility to handle both broadband noise and nonlinear problems [29], as well as non-stationary background flows. 


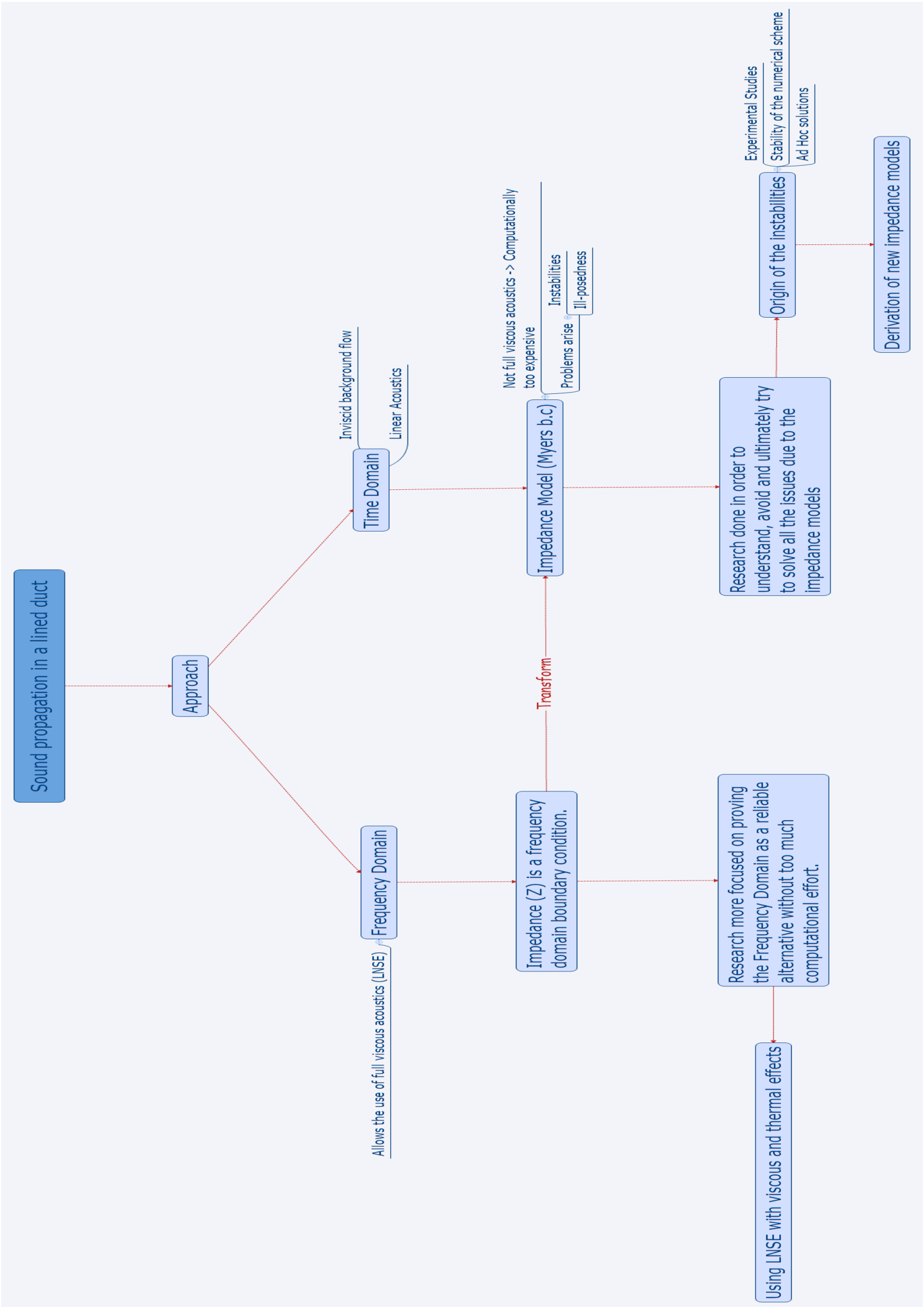

Figure 1.2: Summary of the literature review. 
Traditionally, numerical approaches have disregarded the effects of viscosity since very high Reynold number flows are considered. Moreover, the problem allows linearisation since nonlinear effects are only crucial at the tip of the fan when a supersonic flow is present [25]. Therefore, the sound propagation is predicted using the Linearized Euler Equations (LEE). The macroscopic properties of the locally reacting wall are included in a variable called impedance $(\mathrm{Z})$. In the literature several methods to compute this parameter are found. A brief description of the techniques considered in this thesis are included in Chapter 3.

This initial approach was proved to be insufficient due to several experimental studies confirmed the need to consider viscosity in the calculations [4] [31]. Consequently, models were modified by dividing the flow field in two areas: a core zone where inviscid flow is assumed, and a very small region close to the wall where viscosity is considered. This region is modelled inside a boundary condition that accounts for the near wall effects. This is known as the Myers, or Myers-Ingard, impedance boundary condition and it comes from matching fluid and solid normal displacement [17]. Nonetheless, this combination was proved to be ill-posed and to give rise to instabilities when considering a time domain [24]. From that moment on researchers have been focused on explaining the origin of the instabilities and to find new formulations that are well-posed and capture properly the viscous acoustics.

At the beginning the instability issues were thought to be mainly a numerical issue caused by the use of not fine enough meshes [24]. Apart from refining the mesh, the addition of filtering was a common solution [29]. Richter [23] studied a 3-point filtering method. However, his findings indicated that this process had to be done very cautiously since it varies with every case. Parameters such as grid resolution, the numerical scheme, the liner impedance and the flow velocity have a great impact. But there is a main controversy with this solution since the addition of filtering might difficult the prediction of the sound attenuation.

Nevertheless, it was shown both experimentally and numerically that under certain circumstances the case of a flow over a lined duct can give rise to flow instabilities [10]. Moreover, [3] [31] presented evidence that the assumptions adopted at the boundaries by Myers, when deriving his boundary condition, are not completely right. They pointed out that viscous effects as well as turbulence can alter these conditions. Hence, according to their findings, the continuity of normal displacement assumed by Myers, only holds when the acoustic boundary layer thickness is much smaller than the hydrodynamic boundary layer thickness, which typically occurs in high frequencies. On the other hand, for low frequencies, the continuity of mass velocity normal to the liner would be a more realistic approach.

The Myers impedance boundary condition is used also in impedance eduction processes. Thus, the findings by Aurégan [31] and Brambley [3] highlighted the necessity of checking whether the methodologies used at that time were adequate. To 
that purpose, a new experimental study was done [30]. In that study the measurement of the wavenumber and the scattering matrix was performed on both sides, i.e. for a source propagating upstream and for another one propagating downstream of the test section containing the liner. This measurements would be compared against data computed for a case with uniform mean flow and Myers boundary condition. Both the estimated and the measured axial wavenumbers were used to estimate the effective impedance. In principle, if the impedance model is fully capturing the viscous acoustics, both measurements should offer the same results. This was the case when data was analysed assuming no flow inside the duct. Results from measurements without flow where as expected and a close agreement was found between both directions of propagation. On the contrary, when considering a flow with Mach number of 0.2 , significant discrepancies between both directions were found. Therefore, as many researchers already suspected, the Ingard-Myers boundary condition was not properly capturing the viscous acoustics, and hence, it was proved not to be a suitable impedance eduction method either. Therefore, the development of new boundary conditions that solve the ill-posedness and that properly capture the viscous acoustics in the time domain was justified.

Brambley has been one of the most active researchers developing new boundary conditions that take into consideration viscous and thermal effects. His research activity has been focused on studying the origin of the instabilities and apply that knowledge to the development of new impedance boundary conditions that capture better the physics [5]. In [10] the concept of the instabilities being a numerical issue caused by the usage of a not fine enough mesh is refused. He found out that the instability of the continuous model is not bounded, whereas in the numerical model the instability is bounded. Indeed, it has a maximum value that is affected by the mesh resolution. Hence, fine meshes will have greater maximum values than coarse meshes; however, the instability is still present. Another important result is the fact that the numerical model is absolutely unstable which means that the instability will spread all over the domain. On the other hand, the real boundary layer is found to be convectively unstable, thus, growing only in space.

It is possible to find in the literature several works where the applicability of various impedance boundary conditions is analysed [11]. One of the most thorough studies is presented in [6] where the validity of using inviscid linearized flow together with a boundary condition to include the near-wall effects is discussed. In order to do that a comparison between the three most used impedance boundary conditions are compared with the full Linearised Navier Stokes equations. Important conclusions are reached. Although the assumption of using inviscid fluid with a boundary condition is validated, the error estimation is around $10 \%$ for low frequencies. Furthermore, a trend was observed indicating that this error increases with increasing Mach number and with low frequencies. However, the error with increasing Mach number was found to be less severe. In addition, results presented very low errors outside the boundary layer which validates the hypothesis of using uniform slipping 
inviscid flow instead of modelling the viscous boundary layer. When studying the different impedance boundary conditions it is found that none of them fully capture the behaviour of viscous acoustics. Firstly, the Myers condition, which is known to be ill-posed, was found to be deficient when predicting the effective impedance apart from small regions close to the surface. Therefore, it is not suitable to predict the stability of surface modes but, due to its simplicity, its prediction of acoustic cut-off modes is good. Secondly, the modified Myers condition, despite being well-posed and, thus, appropriate for stability of surface mode predictions, it cannot be trusted since it is derived assuming inviscid flow and, according to the author's findings in that paper, viscosity plays a major role in such studies. Finally, the leading order viscous condition, shown to be ill-posed, was studied and, thus, not adequate for stability studies. However, it offers an improvement in the prediction of upstreampropagating cutoff modes. As a conclusion, it was shown that none of the current impedance boundary conditions is good enough to fully capture the behaviour of viscous acoustics as done by the Linearized Navier-Stokes equations (LNSE). In order to solve this problem, a new impedance boundary condition was derived by Brambley. It seems to agree well with the full numerics but further investigation is still needed.

The second approach shown in Fig.1.2 is the frequency domain. In this case the full LNSE are usually considered. [15] is the first study where time and frequency domain solutions are validated using a nominal impedance. The results obtained with the LNSE are in close agreement with the experimental data presented in [14]. Furthermore, the LNSE in the frequency domain were used to study sound propagation in other applications concerning ducts [2]. The obtained results were again in very close agreement with the experimental data, verifying this methodology as a valuable way of obtaining reliable data compared to other more computationally expensive methods [24].

\subsection{Objectives}

In the previous section it is possible to see that most of the work done by the majority of research groups is in order to find new impedance boundary conditions that capture the viscous acoustics. Some of the results presented justify this effort. However, this problem could also be solved using a frequency domain approach. Some advantages over a time domain are: a serious reduction of the computational effort and the suppression of instabilities as no temporal growth can occur. Moreover, it is worth noting that the impedance boundary condition can be directly applied in the model. Thus, frequency domain models can be a good tool to obtain fast and accurate solutions with a relatively low computational effort. The inability of the Myers impedance boundary condition, to fully capture the viscous behaviour close to the wall, demands finding a different approach where no assumptions close to 
the walls are needed. Such approach is achieved when the Linearised Navier Stokes equations (LNSE) are used. Therefore in this thesis work the LNSE equations will be solved in the frequency domain.

The first part of the thesis will be focus in developing a reliable computational model. The obtained results will be validated against the ones presented in [14]. In the second part, a model with two sources, upstream and downstream of the test section, will be developed. The goal is to measure the axial wavenumbers and the scattering matrix and compare the obtained measurements with the results presented in [30]. The main motivation for this study, is whether in a frequency domain solution the viscous acoustics are fully captured, and therefore, it is a more suitable method than the time domain in order to predict sound propagation in an acoustic liner.

Another important aspect when studying the acoustics of a lined duct is the resolution of the acoustical boundary layer. In the literature review it has been found several mentions to the limitations that arouse when it is included in the computational models. It is obvious that, in a 3D model, the inclusion of the acoustical boundary layer will give raise to the number of total cells which lead to higher computational time and effort. Therefore, it is a fair question to study how resolved the acoustical boundary layer needs to be in order to find good solutions. To the author's best knowledge this question has not been specifically tackled yet. Hence, this is the second study carried in this thesis work. Due to time considerations, only a $2 \mathrm{D}$ case is be treated, but still it could give a trend of the resolution needed to obtain accurate and reliable results that fully capture the viscous acoustics in more complex geometries. 



\section{Chapter 2}

\section{Fundamentals of fluid dynamics}

Fluid dynamics is the branch of science that studies moving and stationary liquids and gases. Its importance is remarkable in fields such as transportation, power generation or civil infrastructure among others. In this chapter some fluid dynamic concepts, that will be used in the present study, are briefly reviewed. For a more detailed view please refer to books such as [19] and [21].

\subsection{Governing Equations}

The motion of fluid is describe by the Navier-Stokes equations (2.1). These set of equations arise from the conservation of mass and momentum combined with stresses due to viscosity and a term accounting for the pressure.

$$
\begin{gathered}
\frac{\partial \rho}{\partial t}+\frac{\partial}{\partial x_{i}}\left(\rho u_{i}\right)=0 \\
\rho \frac{D u_{j}}{D t}=-\frac{\partial p}{\partial x_{j}}+\rho g_{j}+\mu \frac{\partial^{2} u_{j}}{\partial x_{i}^{2}}+\left(\mu_{v}+\frac{1}{3} \mu\right) \frac{\partial}{\partial x_{j}} \frac{\partial u_{m}}{\partial x_{m}}
\end{gathered}
$$

where $u_{i}$ is the velocity component, $\rho$ is the density, $p$ is the pressure, $g_{j}$ represent body forces and $\mu$ is the viscosity.

In some cases the density fluctuation is very small and it can be assumed to be constant, and thus, Eq 2.1 can be reduced to Eq 2.2, which are known as the incompressible Navier-Stokes equations:

$$
\begin{gathered}
\frac{\partial u_{i}}{\partial x_{i}}=0 \\
\rho \frac{D u_{j}}{D t}=-\frac{\partial p}{\partial x_{j}}+\rho g_{j}+\mu \frac{\partial^{2} u_{j}}{\partial x_{i}^{2}}
\end{gathered}
$$


In addition, the momentum equation $(2.2 \mathrm{~b})$ can be further reduced if viscous effects are considered negligible. This is usually true away from the boundaries of the flow field.

$$
\rho \frac{D u_{j}}{D t}=-\frac{\partial p}{\partial x_{j}}+\rho g_{j}
$$

The resultant equation (2.3) is known as the Euler equation.

Note that in both the compressible and incompressible formulation of the NavierStokes equations the energy equation is not included. This is due to the neglection of thermal effects in the scope of this thesis. Therefore, the flow field is assumed to be isothermal and, consequently, a constant value for the temperature is assumed.

\subsection{Boundary Layer}

At the beginning of the twentieth century the most used methodology to solve fluid flow fields was the usage of the Euler equations of motion [28]. These procedure provided decent results for very simple cases, however, when more complicated flow fields were analysed the deviation from the experimental results was obvious. It was known before Prandtl that the consequence of this deviation was due to not considering viscosity in the equations for fluid motion. Nonetheless, it was Prandtl the first one in proposing a solution that had a very close agreement with the experimental data for low-viscosity fluids. According to this theory, viscosity can be neglected everywhere but near the boundaries of the flow field since the no-slip boundary condition must hold. Therefore, this small region where the effect of viscosity is not negligible is known as boundary layer.
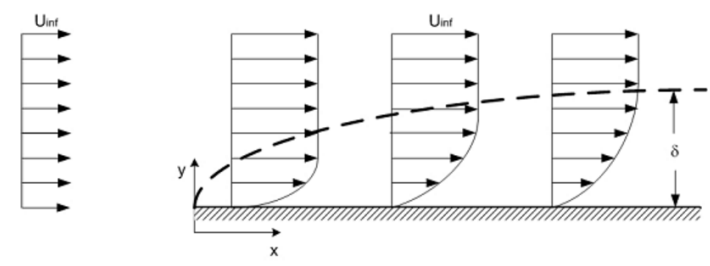

Figure 2.1: Schematic of the boundary layer thickness over a flat plate [28].

In Fig 2.1 a velocity profile close to a wall is shown. The purpose of this image is to illustrate the concept of boundary layer. In front of the leading edge there is a uniform mean flow and it can be seen that the thickness of the boundary layer $(\delta)$ increases as it moves further downstream. This thickness refers to the distance from 
the wall where the streamwise velocity component inside the boundary layer is $99 \%$ of the free-stream velocity component in the streamwise direction. Therefore, this example helps to explain why for flows with low viscosity the flow field can be divided in two zones in order to facilitate its mathematical analyses. Firstly, a region far from the wall where the effect of viscosity can be disregarded and where Euler equations can provide a good approximation. Finally, the second zone is located in the near-wall region, where the effect of viscosity is not negligible and shear stresses must be taken into account.

\subsection{Turbulence}

Turbulence is a flow regime that is characterized by high Reynolds numbers and unpredictability since it cannot be determined by deterministic or analytical analysis as well as three-dimensionality. In such flows, velocity and pressure fluctuate chaotically around a mean value both in time and space. In addition, a large range of vortical structures, known as eddies, are present which increase the diffusivity. Turbulence can be either good or bad, for example, it is usually enhanced in internal combustion engines or chemical reactors since it increases the efficiency of the mixing process. However, this will also lead to higher noise levels which, in turn, is never a desirable feature in almost any modern application.

\subsubsection{Reynolds Average Navier Stokes Equations}

The unsteady Navier-Stokes equations describe succesfully the nature of turbulent flows. If these set of equations is numerically solved all the mean flow and turbulent fluctuations would be determined. However, to carry a direct numerical simulation (DNS) is very computationally demanding since all the length scales, from the large eddy up to the Kolmogorov one, are solved. This is the reason why DNS is not commonly used in industrial problems [13]. A less computationally expensive approach is done by using the Reynolds Average Navier-Stokes equations. In this set of equations, the main flow quantities are obtainend as well as the effects introduced by turbulence. To that purpose, both velocity and pressure are split into two variables accounting for the mean and fluctuating part respectively. This is known as the Reynolds decomposition (2.4),

$$
\begin{aligned}
u_{i} & =U_{i}+u_{i}^{\prime} \\
p & =P+p^{\prime}
\end{aligned}
$$

where ' denotes the fluctuating quantity.

This decomposition can be applied either to Eq 2.1 or Eq 2.2 depending whether a compressible or incompressible flow is considered. In this case, it has been applied to Eq 2.2, that are afterwards time averaged, and the resulting system of equations 
is shown in Eq 2.5, where Eq 2.5b is known as the Reynolds average momentum equation.

$$
\begin{gathered}
\frac{\partial\left(U_{i}+u_{i}^{\prime}\right)}{\partial x_{i}}=0 \\
\frac{\partial U_{i}}{\partial t}+U_{j} \frac{\partial U_{i}}{\partial x_{j}}=-\frac{1}{\rho} \frac{\partial P}{\partial x_{i}}+\frac{\partial}{\partial x_{j}}\left(\mu \frac{U_{i}}{\partial x_{j}}-\overline{u_{i}^{\prime} u_{j}^{\prime}}\right)
\end{gathered}
$$

The main difference between Eq 2.5b and Eq 2.2b is the term known as Reynolds stress $\left(\overline{u_{i}^{\prime} u_{j}^{\prime}}\right)$ which needs to be modelled in order to be solved. Indeed, this system of equations bring an additional problem since they have more unknowns than equations. Hence, modelling is needed in order to be able to solve the Reynolds Average Navier Stokes equations (RANS).

\subsubsection{Turbulence models}

The most widely used turbulence models are known as eddy-viscosity models. The eddy, or turbulent, viscosity is defined such as

$$
\nu_{T} \sim \Lambda V
$$

and it cannot be assumed constant since it depends on the large eddies both length $(\Lambda)$ and velocity $(V)$ scales as shown in Eq 2.6. There are three different types of eddy viscosity models [26]:

- Zero-equation Models: Also known as Algebraic models due to the length and velocity scales are related to the mean flow by means of the velocity gradient, wall distance or thickness of the shear layer. Usually, these models are developed for a specific problem obtaining a good performance.

- One-equation Models: This model typically solve a transport equation for either the turbulence kinetic energy $(K)$ or the eddy viscosity. Either way, the model needs to be completed with additional information from more global conditions, for instance, the distance to the wall. One-equation models usually perform quite well when dealing with attached boundary layers and other types of shear-layer flows. A well-known example is the Spalart-Almaras model which solves the eddy viscosity.

- Two-equation Models: These models solve for two quantities by means of their transport equation. Hence, they are said to be complete since no additional information from global quantities is needed. Two well-known models are the standard $K-\varepsilon$, that solves for the turbulent kinetic energy $(K)$ and its dissipation rate $(\varepsilon)$, and the Menter SST $K-\omega$, that solves the turbulent kinetic energy as well and the turbulence frequency $(\omega)$. Most general CFD softwares include these models since they perform well in a great variety of flows. 


\section{Chapter 3}

\section{Sound propagation in ducts}

Aeroacoustics is the branch of acoustics that deals with sound generated and propagated by the presence of flow. Its utilization is significant to analyse sound generation and propagation in industrial applications such as fans, combustion engines, turbines, flow-structure interaction and jet engines. This chapter introduces the basic theory used to analyse problems concerning sound propagation in a duct. As in Chapter 2, special attention is made to the concepts that are needed for the present study, e.g. dispersion relation in the presence of flow and without flow, governing equations, impedance eduction methods or key concepts for its numerical calculation. For a more detailed view please refer to books such as [20] or [12].

\subsection{Fundamentals of linear acoustics}

Acoustic waves are perturbations that propagate through a flow. Therefore, the equations of motion for a fluid flow still hold. In order to account for this phenomena, a decomposition between a mean and a fluctuating value is introduced, as shown in Eq 3.1,

$$
\begin{gathered}
p(x, t)=p_{0}+p^{\prime}(x, t) \\
\rho(x, t)=\rho_{0}+\rho^{\prime}(x, t) \\
u(x, t)=u^{\prime}(x, t)
\end{gathered}
$$

which are then introduced in the mass and momentum conservation equations as shown in Eq 3.2,

$$
\begin{gathered}
\frac{\partial \rho^{\prime}}{\partial t}+\rho_{0} \nabla \cdot u^{\prime}=m^{\prime} \\
\rho_{0} \frac{\partial u^{\prime}}{\partial t}+\nabla p^{\prime}=f_{v}^{\prime}
\end{gathered}
$$


Note that the ratio $\rho^{\prime} / \rho_{0}$ is very small and so is the velocity associated to the propagation of the wave, $u^{\prime}$, hence, allowing the linearisation of the problem. Therefore, from now on only linear acoustics are considered. In addition, during this derivation it is assumed that no losses, specially due to heat conduction, occur. Hence the fluid is assumed to be isentropic and this condition can be used to eliminate the density from Eq 3.2. The last step is to perform the operation $\partial / \partial t(3.2)-\nabla \cdot(3.2)$ in order to obtain the classical wave equation, shown in Eq 3.3

$$
\frac{1}{c_{0}^{2}} \frac{\partial^{2} p^{\prime}}{\partial t^{2}}-\nabla^{2} p^{\prime}=0
$$

where $\nabla^{2}$ refers to the Laplace operator and $c_{0}$ refers to the isentropic speed of sound.

The simplest solution to Eq 3.3 is achieved assuming only propagation in the x-axis, as presented in Eq 3.4, and is known as plane wave solution.

$$
p^{\prime}(x, t)=p_{+}^{\prime}\left(t-x / c_{0}\right)+p_{-}^{\prime}\left(t+x / c_{0}\right)
$$

where the signs + or - refer to a wave propagating upstream or downstream of the source in the x-axis. In acoustics it is usual to assume a harmonic time dependence and consider the wave field to be complex. Hence, Eq 3.4 can be re-written as shown in Eq 3.5,

$$
p^{\prime}(x, t)=\hat{p}(x) \exp (i \omega t)
$$

where $\hat{p}$ refers to the complex amplitude and $\omega=2 \pi f$ indicates the angular frequency. If this complex pressure is inserted in Eq 3.3, the Helmholtz equation is obtained (Eq 3.6),

$$
\nabla^{2} \hat{p}+k^{2} \hat{p}=0
$$

where $k=\omega / c_{0}$ is defined as the wave number and this definition is valid when no flow is considered. However, in the presence of a background flow the velocity of propagation in the positive direction of the $\mathrm{x}$-axis is higher than in the negative one. To take into account this effect a modification is needed, leading to Eq 3.7 where $k$ now depends on the Mach number. Note that $k_{0}=\omega / c_{0}$ and that the \pm sign refers to a wave propagating upstream or downstream of the source and e refers to the direction of propagation.

$$
k=\frac{k_{0}}{ \pm 1+\mathbf{M} \cdot \mathbf{e}}
$$




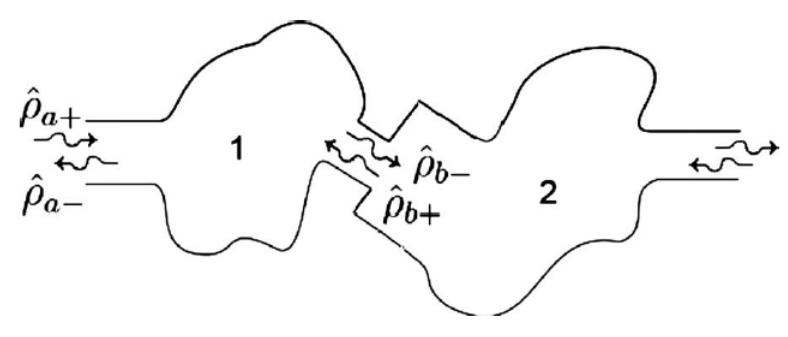

Figure 3.1: Sign criteria used for the calculation of the scattering matrix [2].

\subsubsection{Scattering matrix and plane wave decomposition}

In order to study sound propagation in ducts it is usual to describe the whole system as a network. Generally, it is assumed that only plane waves are propagating in the straight ducts, and thus, a way to determine the sound transmission, if linear theory applies, is the so-called two-port theory. According to [1] a two-port element is defined as a linear physical system with an input and an output, the state of which can be determined by means of two state variables. In particular, if two pressure amplitudes, $\hat{p}_{+}$and $\hat{p}_{-}$, measured at two different cross-sections are used the two-port is said to be in the scattering matrix form.

$$
\left[\begin{array}{c}
\hat{p}_{a^{-}} \\
\hat{p}_{b^{+}}
\end{array}\right]=\left[\begin{array}{ll}
S_{11} & S_{12} \\
S_{21} & S_{22}
\end{array}\right]\left[\begin{array}{c}
\hat{p}_{a^{+}} \\
\hat{p}_{b^{-}}
\end{array}\right]
$$

where $a$ and $b$ are two different cross-sections and the \pm sign refers to the positive and negative direction of propagation which are shown in Fig 3.1. Applying this sign criteria the final system obtained can be written as it follows.

$$
S=\left[\begin{array}{ll}
R^{+} & T^{-} \\
T^{+} & R^{-}
\end{array}\right]=\left[\begin{array}{cc}
\hat{\rho}_{a^{+}}^{I} & \hat{\rho}_{a^{+}}^{I I} \\
\hat{\rho}_{b^{+}}^{I} & \hat{\rho}_{b^{+}}^{I I}
\end{array}\right]\left[\begin{array}{cc}
\hat{\rho}_{a^{-}}^{I} & \hat{\rho}_{a^{-}}^{I I} \\
\hat{\rho}_{b^{-}}^{I} & \hat{\rho}_{b^{-}}^{I I}
\end{array}\right]^{-1}
$$

where $\mathrm{T}$ and $\mathrm{R}$ are the transmission and reflection coefficients respectively and $I$ and $I I$ indicates the cross-section considered. In order to compute the elements it is assumed that the small perturbations can be separated in its upstream and downstream component (Eq 3.8).

$$
\begin{aligned}
& \hat{\rho}=\hat{\rho}_{+}+\hat{\rho}_{-} \\
& \hat{u}=\hat{u}_{+}+\hat{u}_{-}
\end{aligned}
$$

In addition, since only plane waves are considered it can be applied the following relation between the velocity and the density perturbation, $\hat{\rho}= \pm \frac{\rho_{0}}{c_{0}} \hat{u}$, is valid. Therefore, the plane wave decomposition can be written as shown in Eq 3.9, 


$$
\begin{gathered}
\hat{\rho}_{ \pm}=\frac{1}{2}\left(\hat{\rho}_{\text {mean }}+\frac{\rho_{0}}{c_{0}} \hat{u}_{\text {mean }}\right) \\
\hat{\rho}_{\text {mean }}(x)=\frac{1}{H} \int_{0}^{H} \hat{\rho}(x, y) \mathrm{d} y \\
\hat{u}_{\text {mean }}(x)=\frac{1}{H} \int_{0}^{H} \hat{u}(x, y) \mathrm{d} y
\end{gathered}
$$

where mean quantities refer to the variable average over the cross-section studied, as shown in Eq $3.9 \mathrm{~b}$ and Eq 3.9c, and $H$ refers to the duct height. This relation allows the use of just one reference plane, instead of the two needed when only pressure amplitudes are considered, since two different variables are being used.

\subsection{Impedance eduction methods}

Acoustic liners are characterised by its impedance which is a variable that comprehend all the macroscopic properties. Impedance it is defined as the ratio between the acoustic pressure $\hat{p}$ and the velocity normal to the locally reacting wall $\hat{u}$, as shown in Eq 3.10, where $n$ refers to the vector normal to the surface. Note that the impedance is a complex value.

$$
Z(x, \omega)=\frac{\hat{p}(x, \omega)}{\hat{u}(x, \omega) \cdot n}
$$

The impedance can be educed using either experimental techniques or numerical methods. To educe values experimentally is very expensive and hence, several numerical methods have been developed in order to obtain accurate values. This section intends to discuss briefly the most used impedance eduction methods. For a more exhaustive discussion please refer to [16].

The first two methods are based on the Pridmore-Brown equation. Despite both of them consider the boundary conditions for the side walls as rigid and that only zero-order modes occurs in the spanwise direction, they differ when considering the number of modes that are allow to propagate. Single-mode methods assume that there is a dominant mode that propagates trough the liner. An advantage of this method is that it works either with uniform or shear flows. Nonetheless, multimode methods account for more modes propagating through the liner. By using this method it is possible to find reflections and higher-order modes. In order to avoid that, Prony's method is used to obtain a single wavenumber value, used afterwards to determine the impedance, from the multi-modal sound field. 
The last approach is the Finite Element Method applied to the convected Helmholtz equation. An important aspect in this method is the assumption of the liner as a locally reacting wall and the use of the Myers impedance boundary condition to account for the viscous effects. The equations are solved using a continuous Galerkin finite element method. The determination of the impedance value is done minimizing the objective function shown in Eq 3.11.

$$
F(\zeta)=\sum_{i=1}^{N_{p}}\left(p_{\text {num }}\left(x_{i}\right)-p_{\text {data }}\left(x_{i}\right)\right)\left(p_{\text {num }}^{*}\left(x_{i}\right)-p_{\text {data }}^{*}\left(x_{i}\right)\right)
$$

where the superscript ${ }^{*}$ denotes the complex conjugate, $N_{p}$ represents the number of total sample points or microphone locations, $p_{\text {num }}$ is the acoustic complex pressure numerically computed and $p_{\text {data }}$ is the measured acoustic complex pressure.

\subsection{Computational methods for sound propagation in a duct with uniform mean flow}

The majority of sound propagation studies for turbomachinery disregard viscous effects and determine the sound propagation by means of the Linearised Euler equations. However, since this thesis work intends to consider viscous effects during the calculation, the full Linearised Navier Stokes equations (LNSE) are used. In Eq 3.12 the time domain version is presented. Note that despite the domain is considered isothermal the energy equation (Eq 3.12c) is still computed.

$$
\begin{gathered}
\frac{\partial \rho}{\partial t}+\nabla \cdot\left(\rho \overrightarrow{u_{0}}+\rho_{0} \vec{u}\right)=M \\
\rho_{0}\left(\frac{\partial \vec{u}}{\partial t}+(\vec{u} \cdot \nabla) \overrightarrow{u_{0}}+\left(\overrightarrow{u_{0}} \cdot \nabla\right) \vec{u}\right)-\nabla \cdot \sigma=F \\
\rho C_{p}\left(\frac{\partial T}{\partial t}+(\vec{u} \cdot \nabla) T_{0}+\left(\overrightarrow{u_{0}} \cdot \nabla\right) T\right)+\rho C_{p}\left(\overrightarrow{u_{0}} \cdot \nabla\right) T_{0} \\
-\alpha_{0} T_{0}\left(\frac{\partial p}{\partial t}+(\vec{u} \cdot \nabla) p_{0}+\left(\overrightarrow{u_{0}} \cdot \nabla\right) p\right)-\alpha_{0} T\left(\overrightarrow{u_{0}} T\left(\overrightarrow{u_{0}} \cdot \nabla\right) p\right) \\
-\alpha_{0} T\left(\overrightarrow{u_{0}} \cdot \nabla\right) p_{0} \nabla \cdot(k \nabla T)=\Phi+Q
\end{gathered}
$$

where $M$ refers to a mass source in $K g / m^{3} s, F$ to a volume force source in $N / m^{3}$, $Q$ to a heat source in $W / \mathrm{m}^{3}$ and $\Phi$ to the viscous dissipation function. However to perform this study considering a time domain will result in a high computational cost. Therefore, a Fourier transform is taken, as shown in Eq 3.13, and a frequency domain solution is used in order to reduce the computational cost.

$$
\begin{gathered}
i \omega \rho+\nabla \cdot\left(\rho \overrightarrow{u_{0}}+\rho \vec{u}\right)=M \\
\rho_{0}\left(i \omega \vec{u}+(\vec{u} \cdot \nabla) \overrightarrow{u_{0}}+\left(\vec{u}_{0} \cdot \nabla\right) \vec{u}\right)-\nabla \cdot \vec{\sigma}=F
\end{gathered}
$$




$$
\begin{gathered}
\rho_{0} C_{p}\left(i \omega T+(\vec{u} \cdot \nabla) T_{0}+\left(\vec{u}_{0} \cdot \nabla\right) T\right)+\rho C_{p}\left(\vec{u}_{0} \cdot \nabla\right) T_{0} \\
-\alpha_{0} T_{0}\left(i \omega p+(\vec{u} \cdot \nabla) p_{0}+\left(\vec{u}_{0}\right) p\right)-\alpha_{0} T\left(\vec{u}_{0} \cdot \nabla\right) p_{0} \\
-\nabla \cdot(k \nabla T)=\Phi+Q
\end{gathered}
$$

where $M$ indicates a mass source in $K g / \mathrm{m}^{3} \mathrm{~s}, F$ refers to a volume force source in $N / m^{3}, Q$ to a heat source in $W / m^{3}, \Phi$ to the viscous dissipation function and $\omega$ represents the angular frequency. The next step is to model the acoustic absorption process done by the liner. The generalised approximation is to use a time domain and hence, a time domain version of the impedance boundary condition is needed. In addition, this modified boundary condition also includes the near-wall effects caused by the boundary layer. It is known as the Myers impedance boundary condition, Eq 3.14, and as already has been discussed it is ill-posed and give rise to instabilities.

$$
\vec{n} \cdot \vec{u}-\frac{p}{Z}-\vec{u} \cdot \nabla\left(\frac{p}{i \omega Z}\right)+\frac{p}{i \omega Z} \vec{n} \cdot(\vec{n} \cdot \nabla \vec{u})=0
$$

where $\vec{n}$ refers to the vector normal to the wall and $Z$ to the impedance value. However, the impedance is originally a frequency domain boundary condition. Therefore, since a frequency domain is being considered, Eq 3.10 is used to model the acoustic absorption.

It is crucial, when solving numerically sound propagation problems, that the boundaries of the domain are truncated. Otherwise, spurious numerical reflections may arise which could cause the solution to diverge. There are several techniques that can be used to avoid this [25]. The first method is known as the Characteristic method and is achieved by writing the equations in the characteristic form and assume values at the boundaries for the incoming characteristics. In addition, Asymptotic methods can be applied. This technique assumes that the asymptotic pattern of the solution in the far field is already known. The easiest way of doing this in the computational model is by applying the flow characteristic impedance $(\rho c)$ at the boundaries. Another option is to applied Zonal methods. This technique includes a buffer region, i.e. a zone of artificial viscosity that damps the solution.

Another important requirement is to be able to propagate sound waves with lowest numerical error. Therefore, to avoid high dissipation and dispersion errors is important. To that purpose, the number of points per wavelength consider in the simulation becomes an important factor. [9] show that low dispersion errors can be achieved by using a low number of points but fourth order schemes while if a typical second order scheme is used the number of points needs to be increased substantially. Therefore, in this thesis work a mesh sensitivity study is perform in order to find a reasonable choice for the number of points per wavelength and the order of the scheme that provide accurate results with a reasonable computational time. 


\section{Chapter 4}

\section{Methodology}

This thesis work comprises a series of test cases that are run in order to validate a computational model, that will be used afterwards, for the completion of the two study cases proposed. Therefore, in this chapter a description of the geometries, boundary conditions, meshes and computational setup used, in both test and study cases, is included. All the simulations have been done using Comsol Multiphysics 5.2 as solver.

\subsection{Test Case 1: No mean flow}

The two test cases are run to compare the obtained data with the experimental data presented in [14]. To that purpose, a 2D model of the experimental duct is created. The computational model, shown in Fig 4.1, has a length of $0.8128 \mathrm{~m}$ and a height of $0.0508 \mathrm{~m}$. The length of the test section is $0.4064 \mathrm{~m}$. These are the same exact dimensions as the real duct.

The liner is located in the upper wall of the test section. The measurements, however, are taken in the opposite wall and in the same positions where microphones are located in the experiment. This is done in order to emulate the same experimental procedure, and thus, facilitate the subsequent comparison.

The solver uses FEM as discretisation method. In addition, an unstructured mesh is chosen. The mesh, consisting of 50000 elements and an average quality of 0.84 , is developed with a maximum element size of $0.001 \mathrm{~m}$, which generates a very good wave resolution, as shown in Table 4.1. Furthermore, the acoustic boundary layer is captured by the inclusion of 8 layers consisting on quadrilateral elements with a first layer thickness of $4.3 \cdot 10^{-5} \mathrm{~m}$. This value is obtained assuming a frequency of $3000 \mathrm{~Hz}$ which in this case represents the most critical condition. Finally, a growth rate of 1.2 is set for the remaining layers.

The assumption of no flow through the duct simplifies the model, and hence, only 


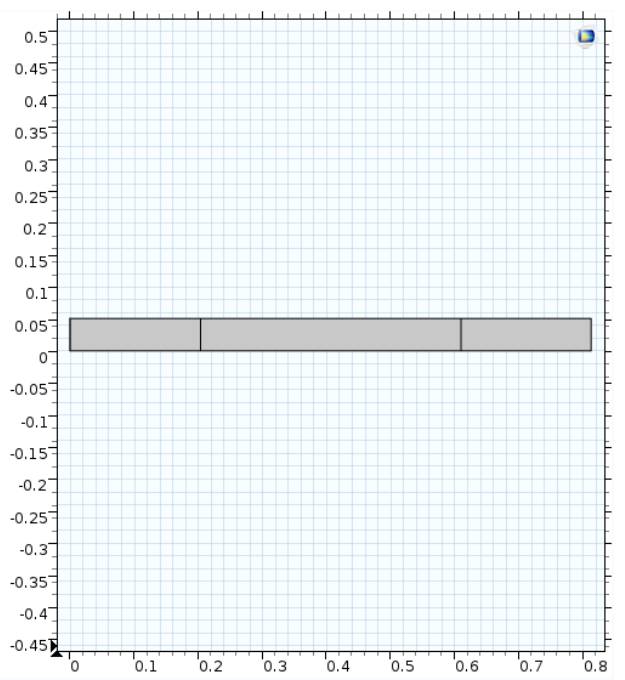

Figure 4.1: Computational domain for Test Case 1

\begin{tabular}{|c|c|}
\hline Frequency [Hz] & Points per wavelength \\
\hline 500 & 689 \\
\hline 1000 & 344 \\
\hline 1500 & 229 \\
\hline 2000 & 172 \\
\hline 2500 & 137 \\
\hline 3000 & 114 \\
\hline
\end{tabular}

Table 4.1: Number of points per wavelength at $\mathrm{M}=0.0$

boundary conditions for the acoustics are needed. In order to generate a sound field a source region is created. Moreover, all the frequencies considered are below cut-on, thus, only plane waves will propagate through the domain. In addition, the source strength, which can be arbitrarily chosen, is set to the value of $2000 \mathrm{~N} / \mathrm{m}^{3}$ in order to obtain a value of $1 \mathrm{~Pa}$ for the pressure amplitude at the inlet. The reason for this procedure is to facilitate the comparison with the analytical solution. Fig 4.2 shows the referred comparison between the numerical and the analytical solution for a plane wave, of amplitude 1, propagating at a frequency of $500 \mathrm{~Hz}$ and 3000 Hz. Note that a good agreement is found.

In this type of analysis it is very important to avoid reflections at the boundaries of the domain. Otherwise, it could cause the solution to diverge due to numerical reflections. There are several methods to prevent this. In this case an asymptotic method was used and it was implemented by applying the characteristic air impedance $\left(\rho_{0} c_{0}\right)$ as boundary condition at both, the domain inlet and outlet. The values used for the liner impedance are shown in Table 4.2 and are obtained from [14]. The rest of 


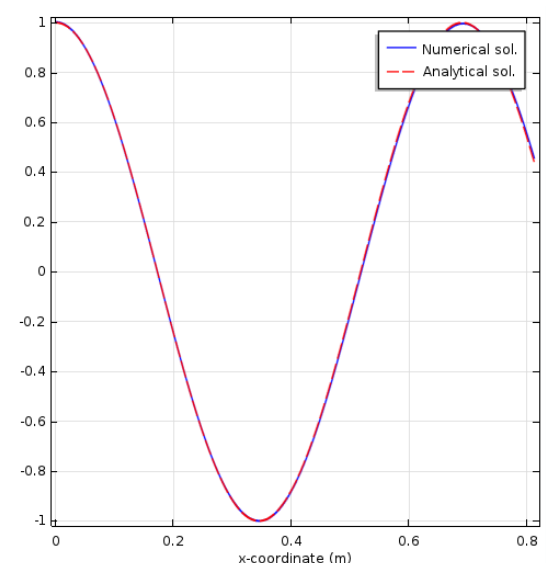

(a) $500 \mathrm{~Hz}$

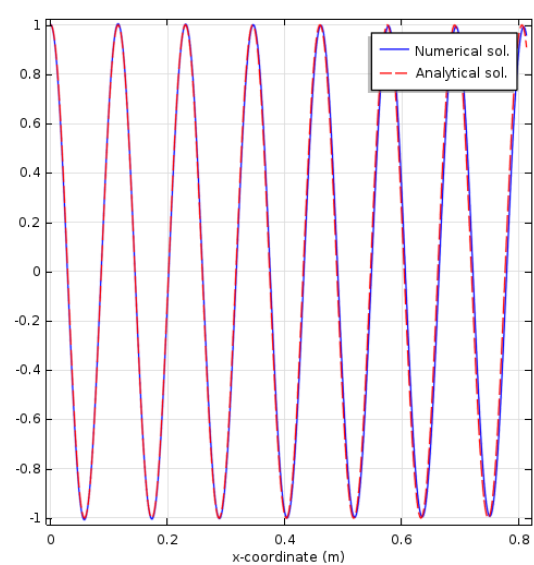

(b) $3000 \mathrm{~Hz}$

Figure 4.2: Comparison between the analytical and the numerical solution for: a) $500 \mathrm{~Hz}$; b) $3000 \mathrm{~Hz}$

the upper and lower walls are modelled using acoustic hard wall boundary condition.

\begin{tabular}{|c|c|c|}
\hline$f(\mathrm{~Hz})$ & $Z_{R} /\left(\rho_{0} C_{0}\right)$ & $Z_{\chi} /\left(\rho_{0} C_{0}\right)$ \\
\hline 500 & 0.51 & -1.68 \\
\hline 1000 & 0.46 & 0.00 \\
\hline 1500 & 1.02 & 1.30 \\
\hline 2000 & 4.05 & 0.62 \\
\hline 2500 & 1.54 & -1.60 \\
\hline 3000 & 0.70 & -0.29 \\
\hline
\end{tabular}

Table 4.2: Normalized liner impedances at $\mathrm{M}=0.0$

Finally, a frequency domain analysis is done for the six frequencies considered using second order schemes for the temperature,the acoustic velocity and the acoustic pressure. In conclusion, these high order schemes together with the high number of points per wavelength should result in a good degree of accuracy.

\subsection{Test Case 2: Uniform mean flow}

In this second test case a uniform mean flow through the duct is included. The use of a hybrid method allows the separation of the model in two steps. The first step will provide a solution for the background flow, whereas in the second step, sound waves are propagated in the already known flow field. 


\subsubsection{Stage I: Background Flow}

In this first stage four different Mach numbers, which values are 0.079, 0.172, 0.255 and 0.335 respectively, are studied. In principle, for Mach numbers lower than 0.3 compressibility effects are negligible, and hence, steady incompressible flow is considered for the first three values. Nonetheless, this assumption is not valid for the last value where a steady compressible flow must be assumed. In addition, all the cases have a turbulent flow regime as the high Reynolds numbers shown in Table 4.3 indicate.

\begin{tabular}{|c|c|}
\hline $\mathrm{M}$ & $\operatorname{Re}_{D}$ \\
\hline 0.079 & $7.5 \cdot 10^{4}$ \\
\hline 0.172 & $1.6 \cdot 10^{5}$ \\
\hline 0.255 & $2.4 \cdot 10^{5}$ \\
\hline 0.335 & $3.2 \cdot 10^{5}$ \\
\hline
\end{tabular}

Table 4.3: Reynolds numbers based on the duct height for all the Mach numbers considered.

The geometry of the computational domain is the same as in the no flow case except for the addition of an extended area in front of the source region, as shown in Fig 4.3 , in order to bring the flow to a fully developed state. The length of that extension varies with increasing Mach number although values oscillate between 70 and 220 duct heights.

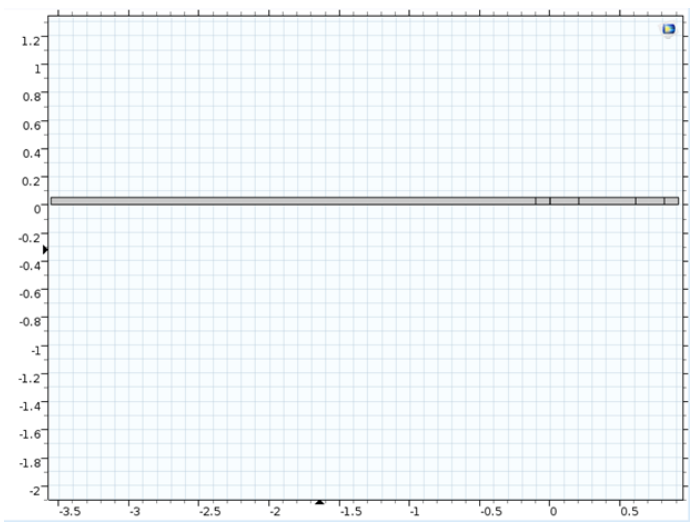

Figure 4.3: Computational domain for Test Case 2

Concerning the mesh, an unstructured one is created. Some mesh parameters, such as the number of total cells or the average quality, are shown in Table 4.4 for every case. Note that since all the incompressible cases have the same extended area the mesh is the same, only varying the Mach number considered. In order to account 


\begin{tabular}{|c|c|c|}
\hline & Incompressble & Compressible \\
\hline Max. Element size $[\mathrm{m}]$ & 0.0025 & 0.004 \\
\hline Number of elements & 163601 & 163101 \\
\hline Average quality per element & 0.77 & 0.79 \\
\hline
\end{tabular}

Table 4.4: Mesh parameters for both compressible and incompressible cases.

for the boundary layer, 8 layers of quadrilateral elements are inserted as well. The first layer thickness is estimated assuming a value of 1 for the $y+$ and a growth rate of 1.2 is established for the remaining layers.

The turbulent model used is the standard $K-\varepsilon$ model. A second order scheme is used for the velocity while first order schemes are chosen for pressure and temperature. This choice represents a compromise between accuracy and computational time. The convergence criteria is set to $10^{-3}$ for the same reason. In addition, a velocity inlet and a pressure outlet are used as boundary conditions. Regarding the walls, a no-slip boundary condition is used in the upper and lower walls. The use of a slip wall for the liner was discarded since it was found in the literature that the prediction of the sound attenuation is not very affected by the wall boundary condition.

\subsubsection{Stage II: Acoustic Propagation}

The computational domain used in this second stage is the same as in the no flow case. Concerning the boundary conditions, acoustic hard walls are applied in the upper and lower walls except for the liner. In that case, the impedance boundary condition is used and the values adopted are shown in Table 4.2. In order to avoid numerical reflections, an absorbing region is created at both sides of the duct. This is achieved by means of a Perfectly Matched Layer (PML), with a length of 0.1016 $\mathrm{m}$, which is the most widely used method in order to truncate the computational domain when open boundaries are considered. The sound field is generated using a source strength, which value varies at every case, in order to keep a value for the pressure amplitude at the inlet equal to $1 \mathrm{~Pa}$.

For the acoustic domain, an unstructured mesh is created with a total number of elements of 69296 and an average quality of 0.91 . All the cases include 8 layers consisting on quadrilateral elements in order to capture the acoustic boundary layer. The value used as the first layer thickness for every case is $4.3 \cdot 10^{-5} \mathrm{~m}$ since it is the most critical case.

A frequency domain analysis is run for the six different frequencies considered. The same frequencies as in the previous test case are studied, thus, no higher order modes are expected to occur neither. Second order schemes are used for the temperature, acoustic velocity and acoustic pressure. 


\subsection{Study Case 1: Viscous effects in a local reacting wall}

In [30] it was proved the failure of the Myers boundary condition. The motivation of this study is, therefore, to use the educed impedance values, and to check whether the obtained results are close to the experimental values, when a viscous domain is considered. To that purpose, a new computational domain is created to match the dimensions of the real experimental apparatus It has a length of $0.495 \mathrm{~m}$ and a height of $0.015 \mathrm{~m}$. The test section has a length of $0.2 \mathrm{~m}$.

The analysis is divided in two stages. During the first stage the flow field is determined. Accordingly, due to a Mach number of 0.2 is studied, an steady state incompressible flow is assumed. A Reynolds number, based on the duct height, of $5.75 \cdot 10^{4}$ indicates that the flow has reached a turbulent state. Hence, the standard $K-\varepsilon$ model is used. Regarding the computational setup, the flow field is solved using a second order scheme for the velocity and a first order scheme for the pressure and the temperature. The boundary conditions used are a velocity inlet, with a value of $68 \mathrm{~m} / \mathrm{s}$, and a pressure outlet with a gauge pressure of $0 \mathrm{~Pa}$. At the walls the no-slip boundary condition is applied. Note that a slip wall is not used at the liner for the same reasons mentioned in the previous section. An unstructured mesh is created with a maximum element size of $1.5 \cdot 10^{-3} \mathrm{~m}$ and an average quality of 0.91 per element. In addition, the boundary layer is completely resolved by adding 8 layers of quadrilateral elements with a growth rate of 1.2 , and a first layer thickness of $0.0021 \mathrm{~m}$ that corresponds to a $y+$ value equal to 1 .

Concerning the acoustic propagation, a mesh sensitivity study is conducted with four different meshes with 13, 23, 68 and 100 points per wavelength respectively. Note that the study is carried for a single frequency of $500 \mathrm{~Hz}$ and that the mesh is assumed to be converged if the variation from the analytical value is less or equal to $5 \%$. The converged mesh is then assumed to work appropriately for the rest of frequencies.

In Fig 4.4 results for only three number of points per wavelength are plotted. It possible to see that, even for the smallest one, the error is less than the permitted $5 \%$ for the majority of the duct. Nonetheless, a mesh with 100 elements per wavelength is finally chosen since it provides better accuracy at high frequencies, being the worst case 13 points per wavelength for a frequency of $3000 \mathrm{~Hz}$. Such number of points per wavelength is usually estimated fine enough for a good resolution. In addition, to obtain a better accuracy the mesh is further optimized obtaining a final relative error lower than $2 \%$ with respect to the analytical solution. The boundary layer is accounted for using 8 layers of quadrilateral elements with a growth rate of 1.2, and a first layer thickness of $4.3 \cdot 10^{-5} \mathrm{~m}$ that corresponds to a frequency of $3000 \mathrm{~Hz}$. 


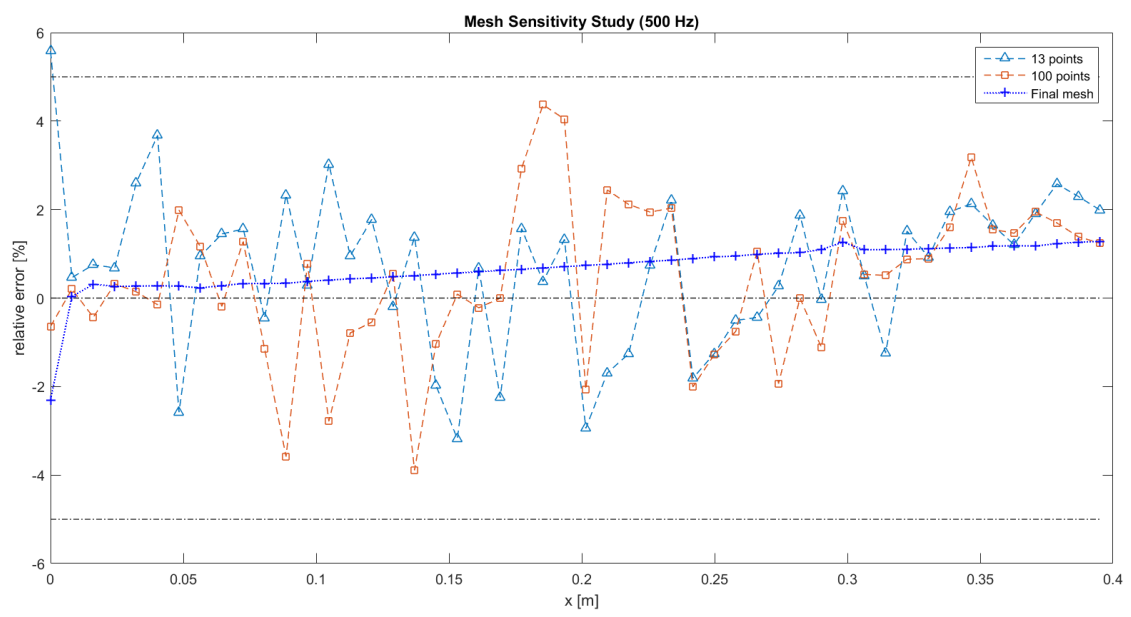

Figure 4.4: Mesh sensitivity study for a frequency of $500 \mathrm{~Hz}$.

The boundary conditions applied are acoustic hard wall to the upper and lower walls except for the liner, where the impedance boundary condition is used. The values are shown in Table 4.5. In addition, two PML, with a length of $48.75 \cdot 10^{-3}$ $\mathrm{m}$, are added in order to avoid spurious reflections at the domain boundaries. Furthermore, all the simulations are done using a third order scheme for temperature, velocity and pressure, which despite the low number of points per wavelength for high frequencies, it should still provide accurate results since 848368 degrees of freedom are solved.

\begin{tabular}{ccccc}
\hline$f(\mathrm{~Hz})$ & \multicolumn{2}{c}{$Z_{R} /\left(\rho_{0} C_{0}\right)$} & \multicolumn{2}{c}{$Z_{\chi} /\left(\rho_{0} C_{0}\right)$} \\
\hline & Upstream & Downstream & Upstream & Downstream \\
500 & 1 & 1 & -1.6 & -3.5 \\
1000 & 0.6 & 0.8 & -0.95 & -1.6 \\
1500 & 0.5 & 0.6 & -0.2 & -0.5 \\
2000 & 0.4 & 0.55 & 0.3 & 0.3 \\
2500 & 0.38 & 0.39 & 0.8 & 0.8 \\
3000 & 0.38 & 0.38 & 1.4 & 1.4 \\
\hline
\end{tabular}

Table 4.5: Liner impedances at $\mathrm{M}=0.2$ extracted from [30]. 


\subsection{Study Case 2: Acoustic boundary layer sensitivity study}

The use of the LNSE forces to resolve the acoustic boundary layer which is generally much smaller than the hydrodynamic one, and hence, it can give rise to an increment of the computational time. Therefore, in this second study the importance of accounting for the acoustic boundary layer is assessed. The same computational setup as in section 4.2 is used. No further modifications are included so that it is clear the effect this parameter has on the final solution. Two different Mach numbers are considered, 0.172 and 0.335 respectively. The acoustic boundary layer, which is the same for both cases since it is frequency dependent as shown in Eq 4.1, is calculated and then it is gradually incremented up to the height of the hydrodynamic boundary layer.

$$
\delta_{a c}=\sqrt{\frac{2 \nu}{\omega}}
$$

where $\nu$ refers to the kinematic viscosity of the flow and $\omega=2 \pi f$ to the angular frequency. The variables studied to assess the impact are the sound pressure level and the phase, that are compared with the experimental values in [14]. 


\section{Chapter 5}

\section{Results and Validation}

This section presents the results for both test and study cases. Note that all the material concerning the test cases performed with Mach number 0.079, 0.172 and 0.255 respectively is shown in Appendix 1. Thus only the results concerning no mean flow and Mach number 0.335 are presented here.

\subsection{Test Case 1}

The contour plots for the acoustic pressure for a frequency of 500 and $3000 \mathrm{~Hz}$ are shown in Fig 5.1. The sound attenuation produced by the liner impedance is significant. In order to effectively compare this attenuation effect with the experimental values, the sound pressure level and the phase are calculated for the same coordinates along the horizontal axis where microphones are located in the experimental study. The sound pressure level (SPL) is defined as a logarithmic measure of the pressure magnitude in relation to a reference value, set to $20 \mu P a$, that corresponds to the lowest hearing threshold of human ear. It is defined as shown in Eq. 5.1,

$$
S P L=20 \cdot \log _{10}\left(\frac{|p|}{p_{\text {ref }}}\right)
$$

On the other hand, all the acoustic values are complex. Hence, the phase, or argument, of a complex number $a \pm b i$ is calculated as shown in Eq. 5.2,

$$
\tan \theta=\frac{b}{a}
$$

where $a$ is the real part and $b$ the imaginary part.

In Fig 5.2 and Fig 5.3, the calculated SPL and phase, are plotted against the experimental values. It can be seen that both, SPL and phase, present a very close agreement for every frequency. Therefore, it can be ascertain that the model provides an accurate solution when no mean flow is assumed. 


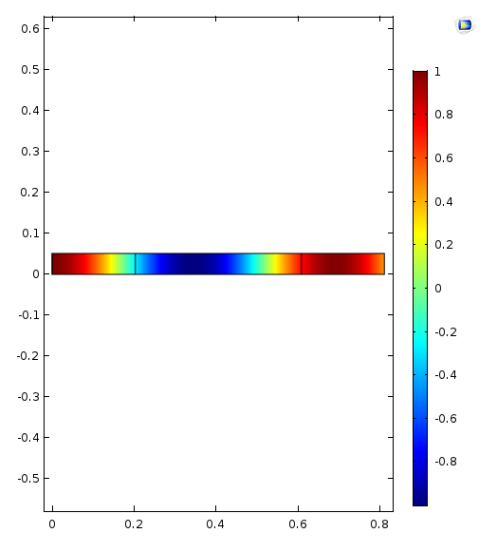

(a) $500 \mathrm{~Hz}$ without impedance.

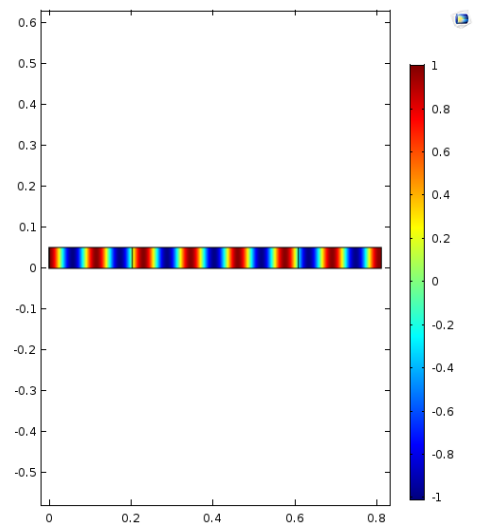

(c) $3000 \mathrm{~Hz}$ without impedance.

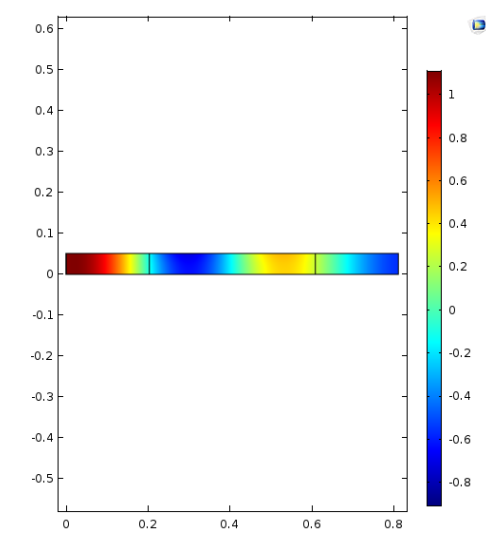

(b) $500 \mathrm{~Hz}$ with impedance.

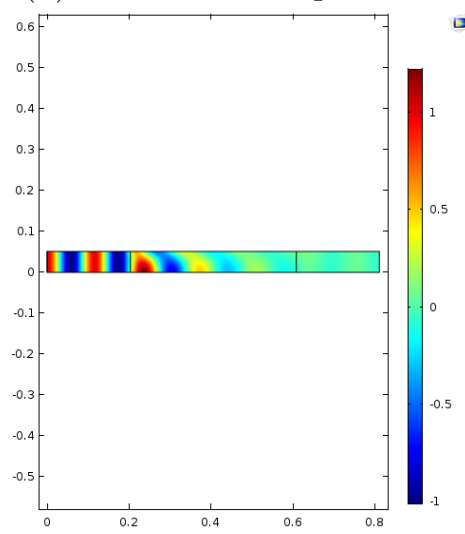

(d) $3000 \mathrm{~Hz}$ with impedance.

Figure 5.1: Pressure contour plots for 500 and $3000 \mathrm{~Hz}$ for Test Case 1.

\subsection{Test Case 2}

In Fig 5.4 and Fig 5.5, the values corresponding to the obtained SPL and phase respectively are presented. Note that the initial condition during the experimental study was to set the value of the SPL at the source plane to a value of $130 \mathrm{~dB}$. This initial condition was not possible to achieve in Comsol, hence, a pressure amplitude of 1 at the inlet of the duct was adopted. Nonetheless, since linear acoustics are considered, the obtained results are scaled up afterwards to match this initial condition. Note that this procedure was applied in every case.

In general a good agreement is found. The behaviour at the lined wall is well captured and the deviation at the exit of the liner is quite small. Regarding the phase, agreement is found to be good again, and the general behaviour is well captured at every frequency. Likewise, the same performance is identified when considering other Mach numbers, attaining always a good agreement in general, for both phase and SPL. Thus, it can be ascertain that the model is capturing the viscous acoustics properly when a uniform mean flow through the duct is assumed. 


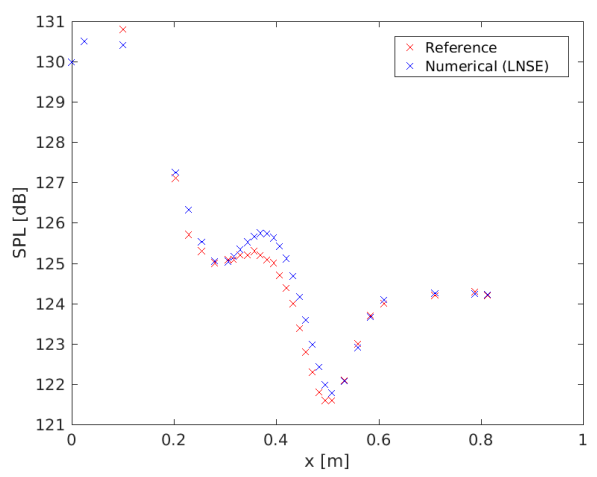

(a) $500 \mathrm{~Hz}$

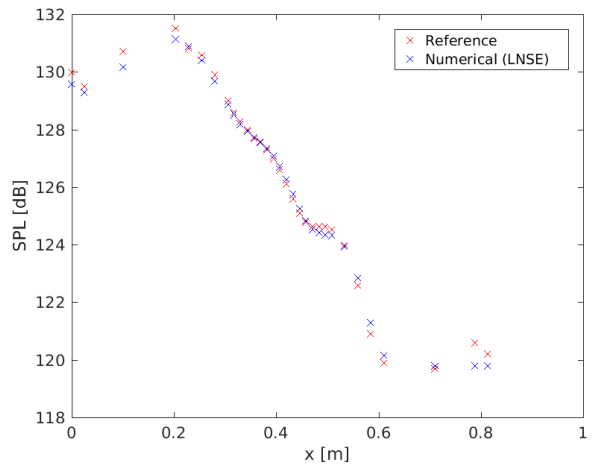

(c) $1500 \mathrm{~Hz}$

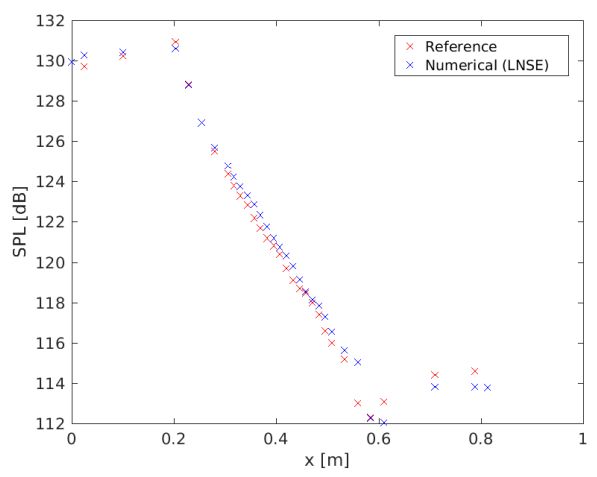

(e) $2500 \mathrm{~Hz}$

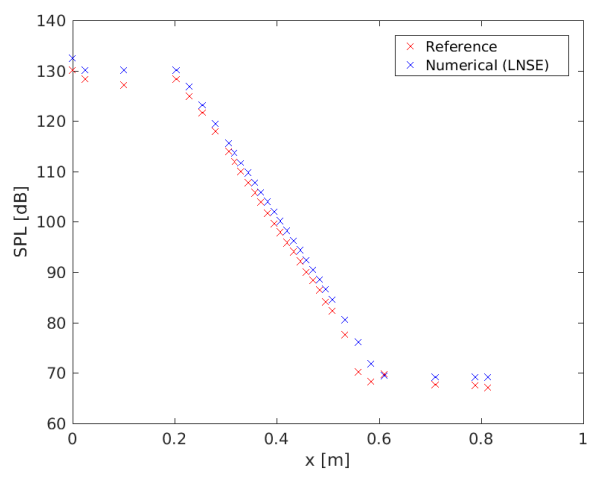

(b) $1000 \mathrm{~Hz}$

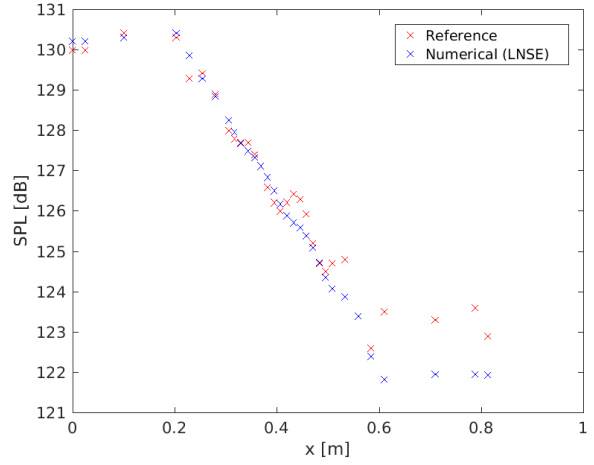

(d) $2000 \mathrm{~Hz}$

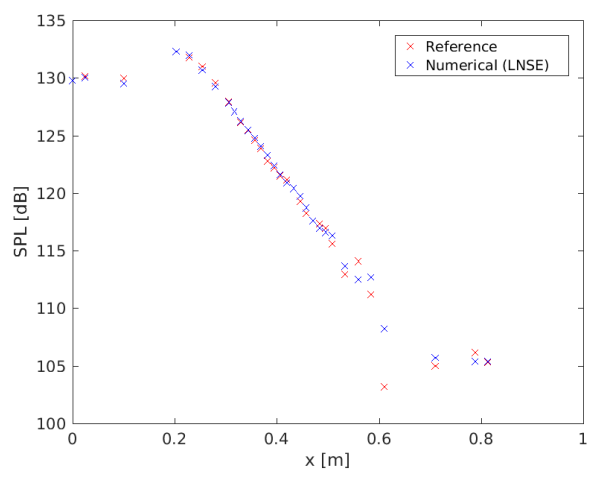

(f) $3000 \mathrm{~Hz}$

Figure 5.2: Measured Sound Pressure Level for Test Case 1 (in blue) versus the reference values (in red). 


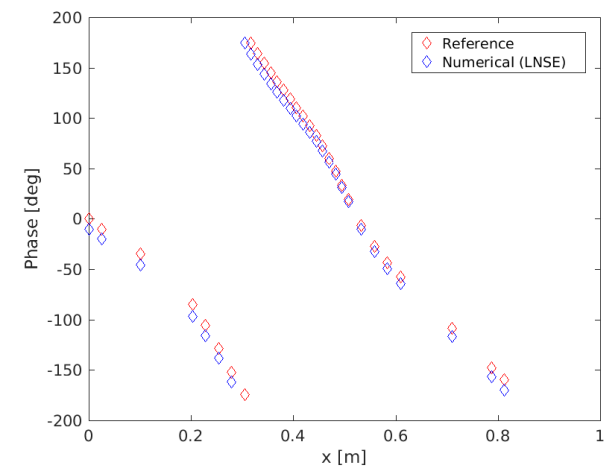

(a) $500 \mathrm{~Hz}$

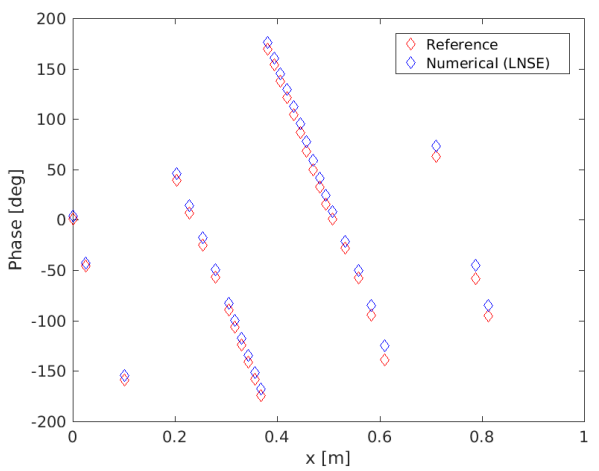

(c) $1500 \mathrm{~Hz}$

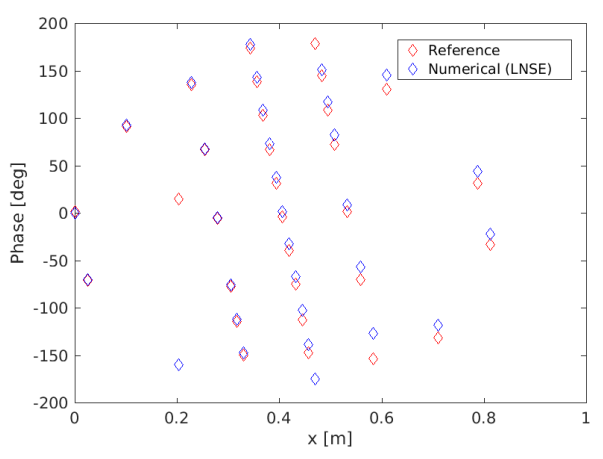

(e) $2500 \mathrm{~Hz}$

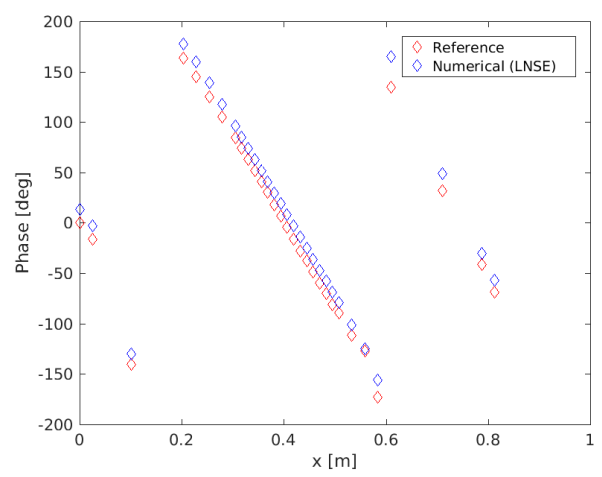

(b) $1000 \mathrm{~Hz}$

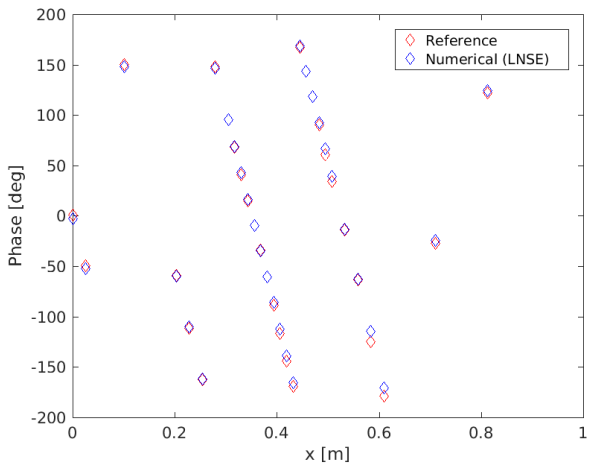

(d) $2000 \mathrm{~Hz}$

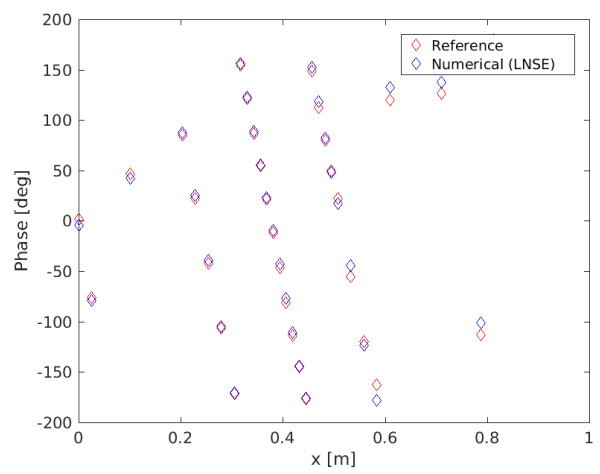

(f) $3000 \mathrm{~Hz}$

Figure 5.3: Phase for Test Case 1 (in blue) versus the phase calculated for the reference values (in red). 


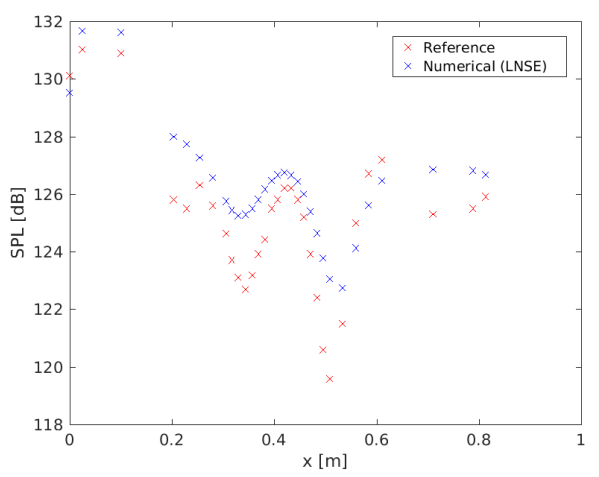

(a) $500 \mathrm{~Hz}$

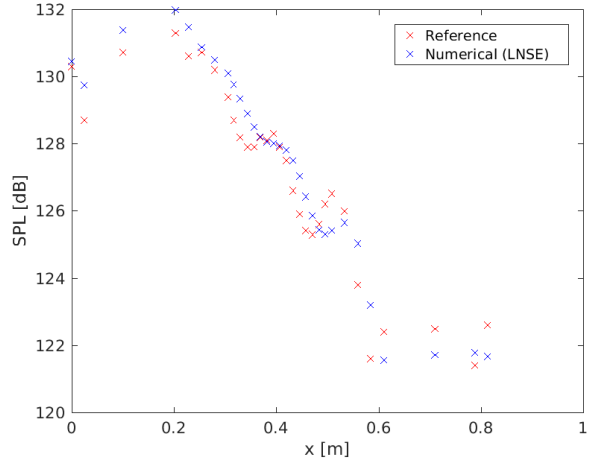

(c) $1500 \mathrm{~Hz}$

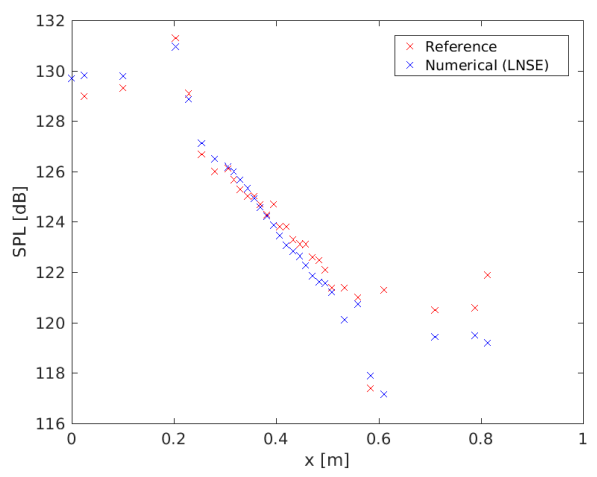

(e) $2500 \mathrm{~Hz}$

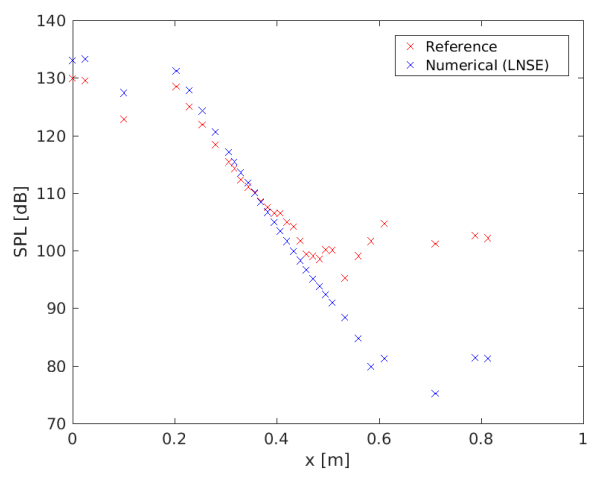

(b) $1000 \mathrm{~Hz}$

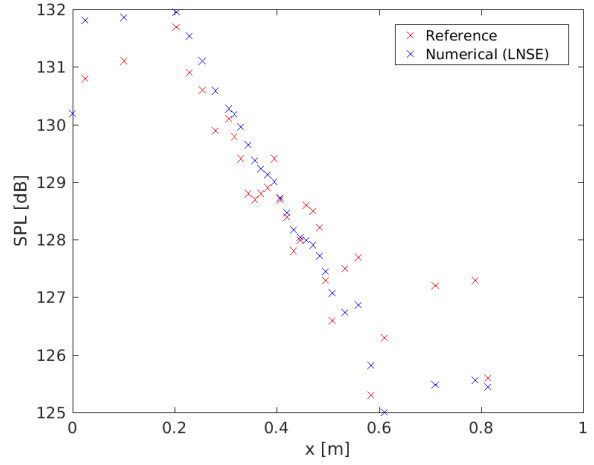

(d) $2000 \mathrm{~Hz}$

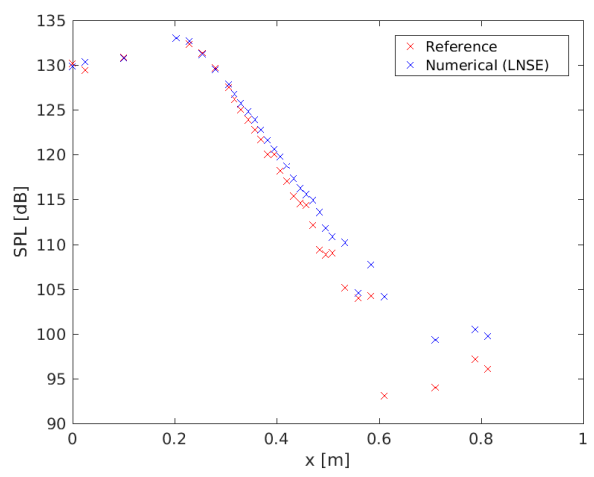

(f) $3000 \mathrm{~Hz}$

Figure 5.4: SPL for Test Case 2 (in blue) versus the phase calculated for the reference values (in red) at $M=0.335$. 


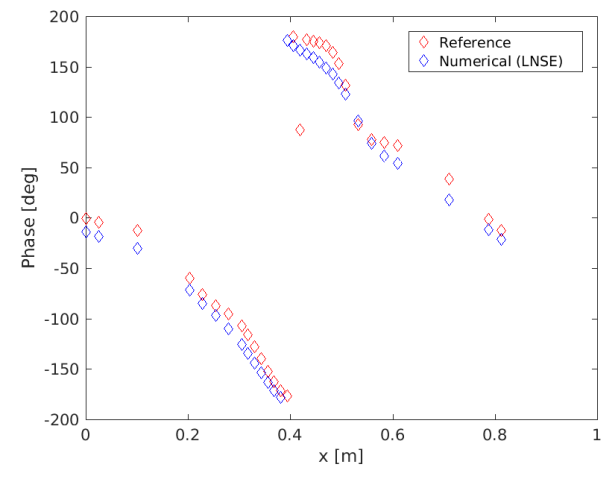

(a) $500 \mathrm{~Hz}$

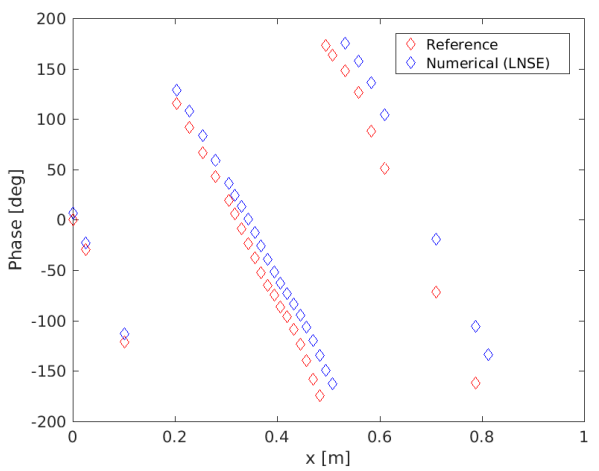

(c) $1500 \mathrm{~Hz}$

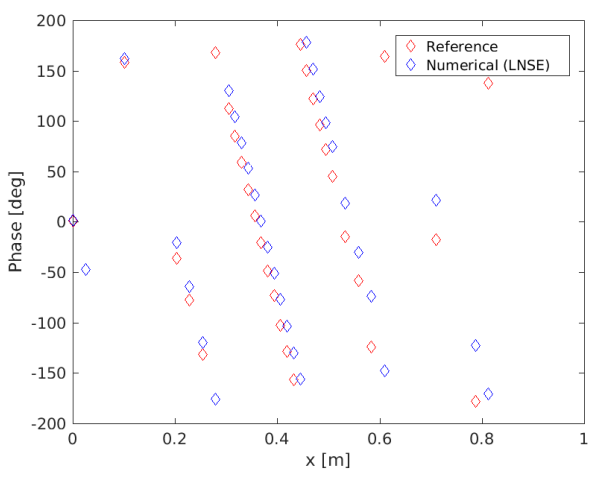

(e) $2500 \mathrm{~Hz}$

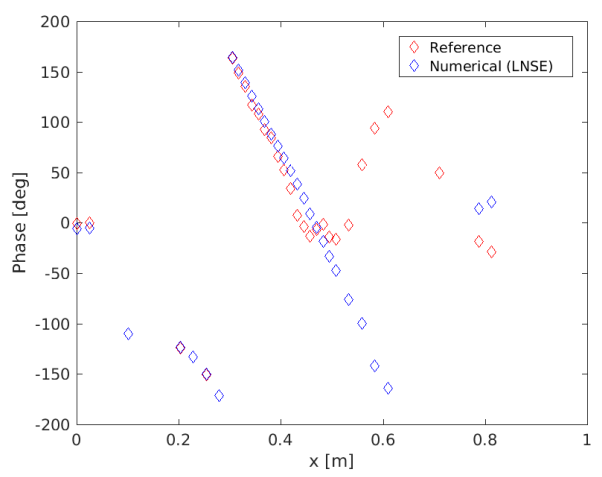

(b) $1000 \mathrm{~Hz}$

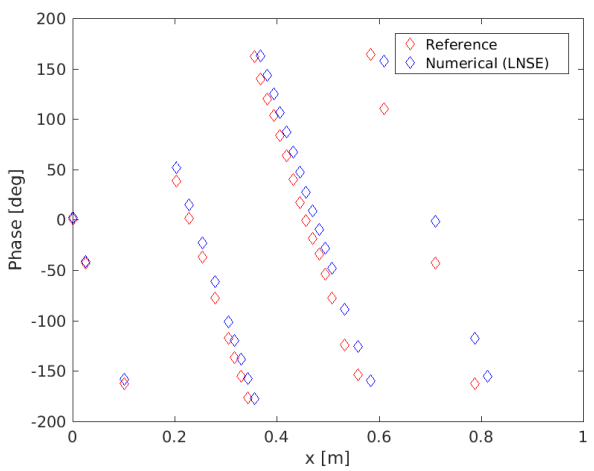

(d) $2000 \mathrm{~Hz}$

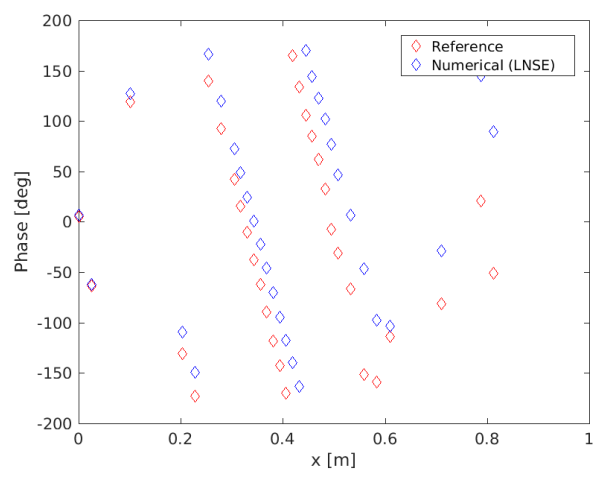

(f) $3000 \mathrm{~Hz}$

Figure 5.5: Phase for Test Case 2 (in blue) versus the phase calculated for the reference values (in red) at $M=0.335$. 


\subsubsection{Impedance sensitivity study}

In chapter 3, several techniques to educe impedances were explained. The aim of this study is, therefore, to validate the model by comparing its performance when impedance values obtained with different techniques are compared. The values used are shown in Table 5.1 and Table 5.2. The SPL is presented in Fig 5.6 and Fig 5.8, whereas the phase is shown in Fig 5.7 and Fig 5.9 for the two Mach numbers considered. The impedance values are obtained from [15] [22] [14].

\begin{tabular}{ccccccc}
\hline$f(\mathrm{~Hz})$ & \multicolumn{3}{c}{$Z_{R} /\left(\rho_{0} C_{0}\right)$} & \multicolumn{3}{c}{$Z_{\chi} /\left(\rho_{0} C_{0}\right)$} \\
\hline & Jones & Burak & O'Reilly & Jones & Burak & O'Reilly \\
500 & 0.51 & 0.62 & 0.57 & -1.68 & -1.66 & -1.60 \\
1000 & 0.46 & 0.48 & 0.47 & 0.00 & 0.03 & 0.00 \\
1500 & 1.02 & 1.09 & 1.08 & 1.30 & 1.23 & 1.30 \\
2000 & 4.05 & 4.38 & 4.68 & 0.62 & 0.88 & 0.77 \\
2500 & 1.54 & 1.50 & 1.55 & -1.60 & -1.55 & -1.63 \\
3000 & 0.70 & 0.72 & 0.72 & -0.29 & -0.30 & -0.27 \\
\hline
\end{tabular}

Table 5.1: Liner impedances at $\mathrm{M}=0.0$

\begin{tabular}{ccccccc}
\hline$f(\mathrm{~Hz})$ & \multicolumn{3}{c}{$Z_{R} /\left(\rho_{0} C_{0}\right)$} & \multicolumn{3}{c}{$Z_{\chi} /\left(\rho_{0} C_{0}\right)$} \\
\hline & Jones & Burak & O'Reilly & Jones & Burak & O'Reilly \\
500 & 0.51 & 0.62 & 0.43 & -1.68 & -1.66 & -0.72 \\
1000 & 0.46 & 0.48 & 0.43 & 0.00 & 0.03 & 0.07 \\
1500 & 1.02 & 1.09 & 1.15 & 1.30 & 1.23 & 1.43 \\
2000 & 4.05 & 4.38 & 5.08 & 0.62 & 0.88 & -2.13 \\
2500 & 1.54 & 1.50 & 1.00 & -1.60 & -1.55 & -1.39 \\
3000 & 0.70 & 0.72 & 0.66 & -0.29 & -0.30 & -0.30 \\
\hline
\end{tabular}

Table 5.2: Liner impedances at $\mathrm{M}=0.335$ 


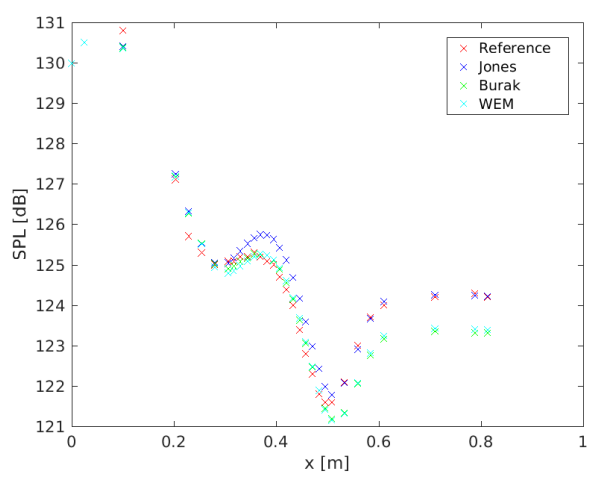

(a) $500 \mathrm{~Hz}$

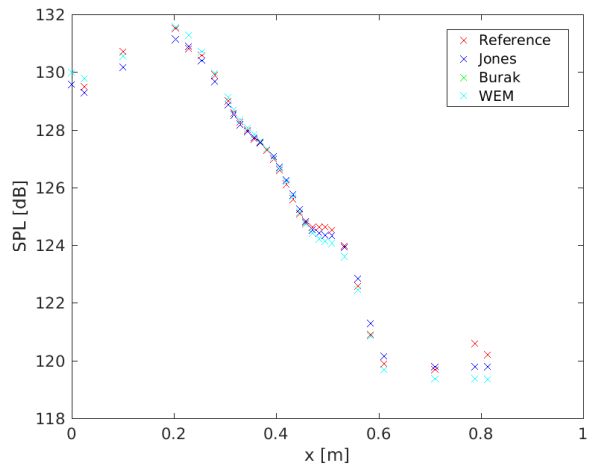

(c) $1500 \mathrm{~Hz}$

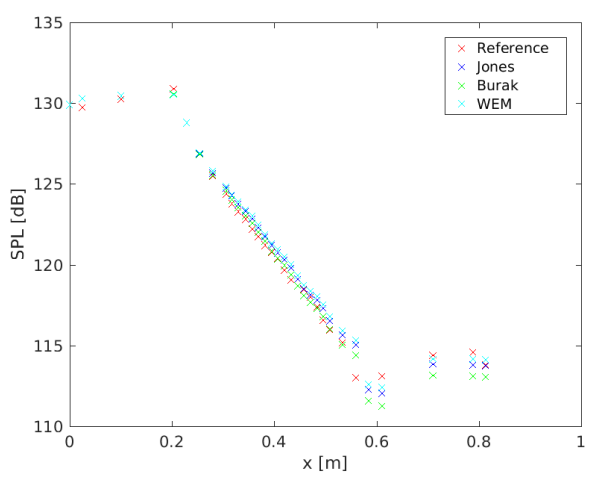

(e) $2500 \mathrm{~Hz}$

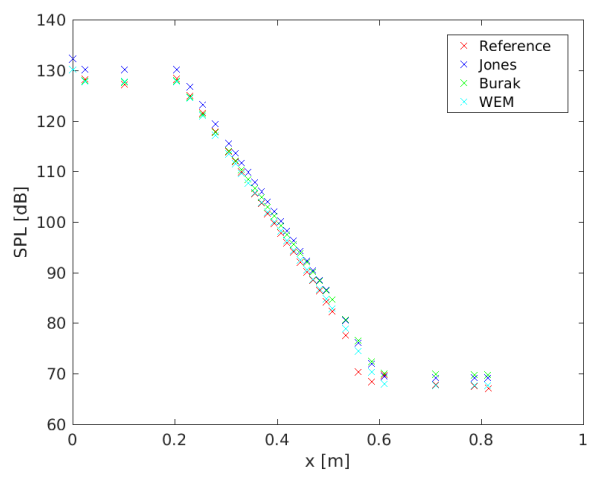

(b) $1000 \mathrm{~Hz}$

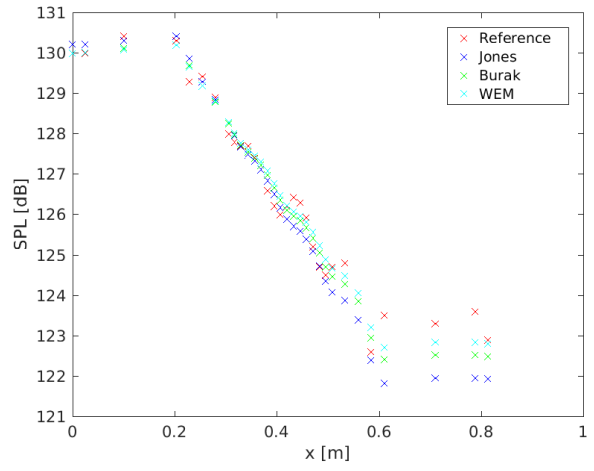

(d) $2000 \mathrm{~Hz}$

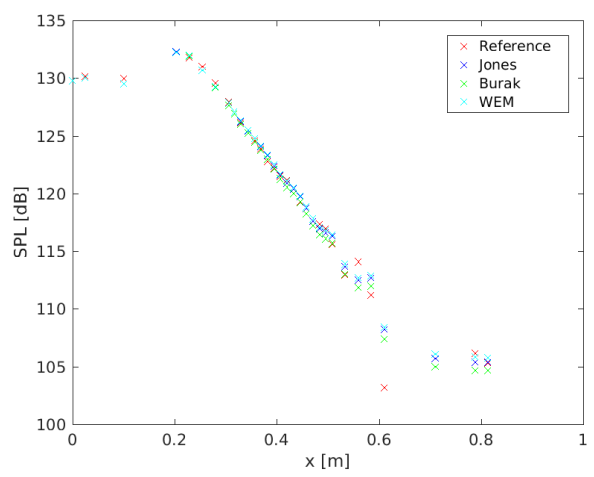

(f) $3000 \mathrm{~Hz}$

Figure 5.6: SPL for the impedance sensitivity study at $M=0$. 


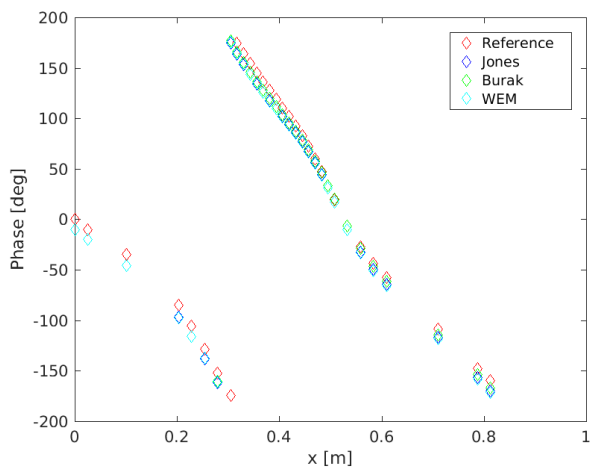

(a) $500 \mathrm{~Hz}$

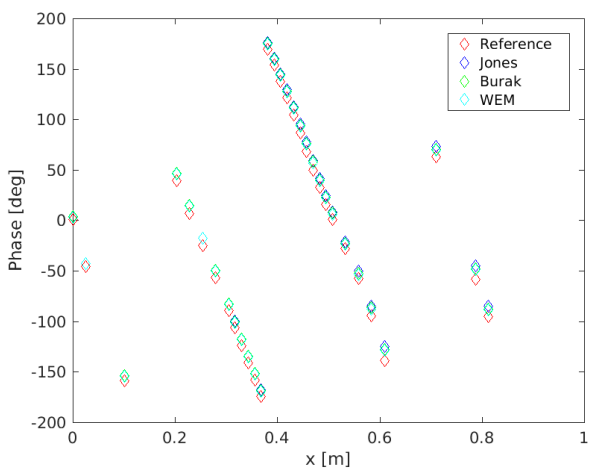

(c) $1500 \mathrm{~Hz}$

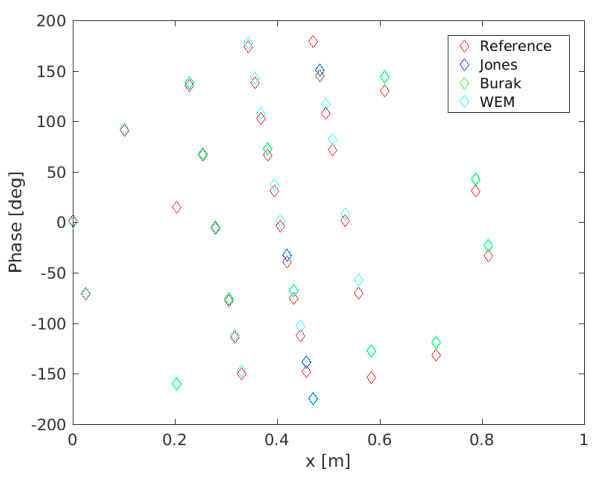

(e) $2500 \mathrm{~Hz}$

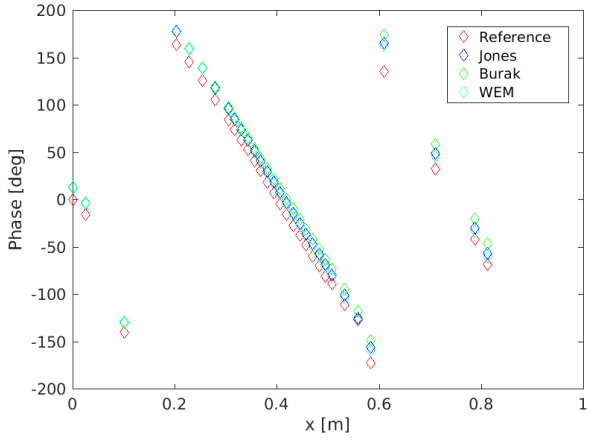

(b) $1000 \mathrm{~Hz}$

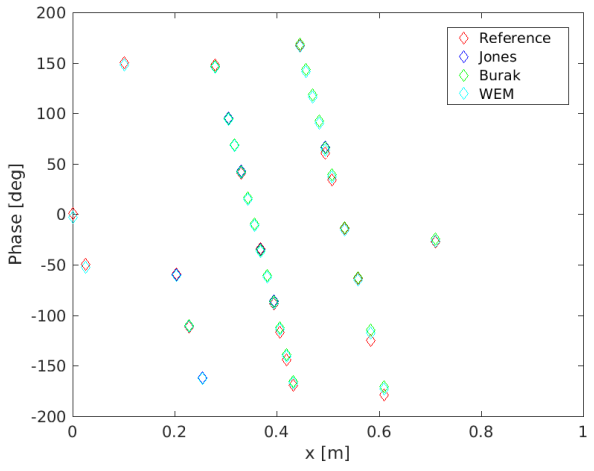

(d) $2000 \mathrm{~Hz}$

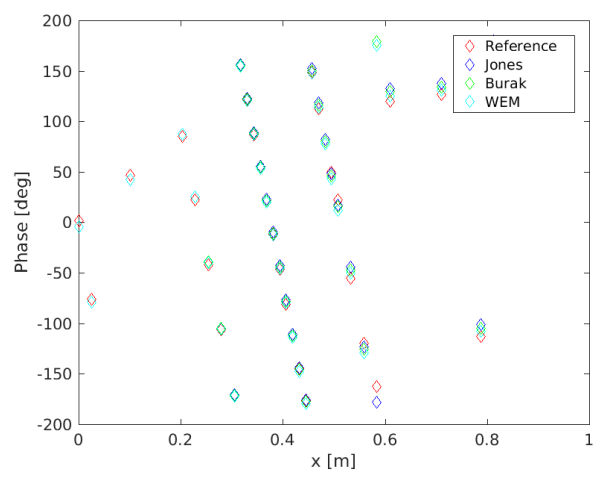

(f) $3000 \mathrm{~Hz}$

Figure 5.7: Phase for the impedance sensitivity study at $M=0$. 


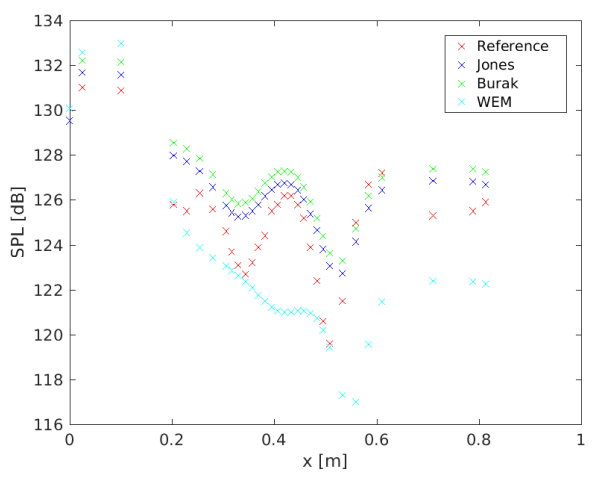

(a) $500 \mathrm{~Hz}$

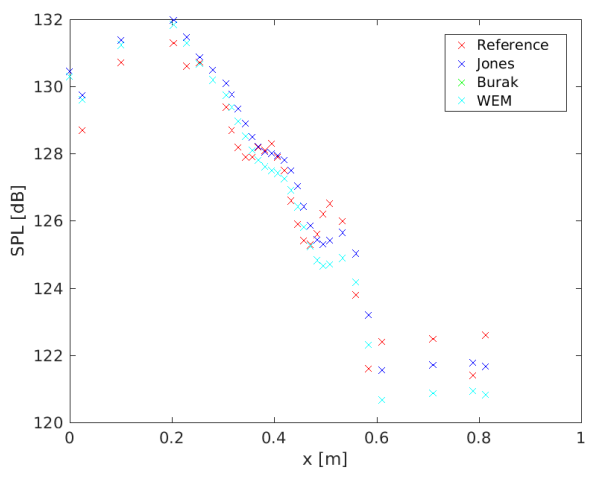

(c) $1500 \mathrm{~Hz}$

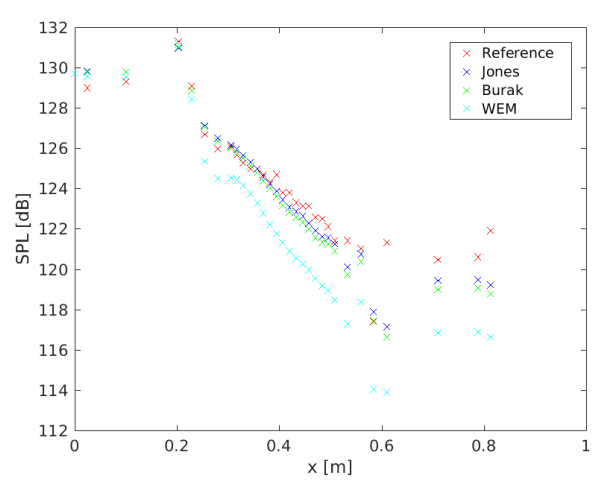

(e) $2500 \mathrm{~Hz}$

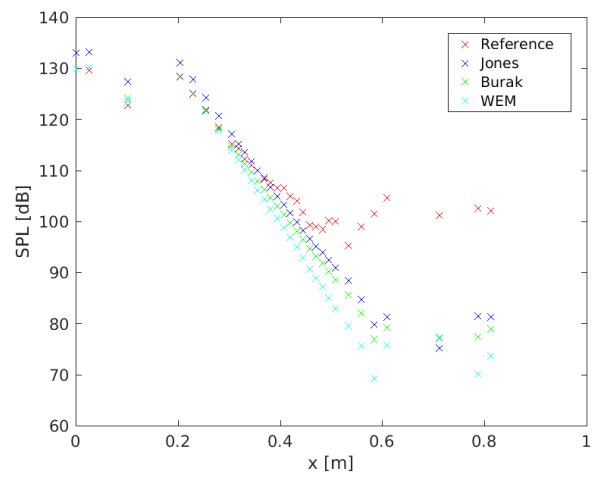

(b) $1000 \mathrm{~Hz}$

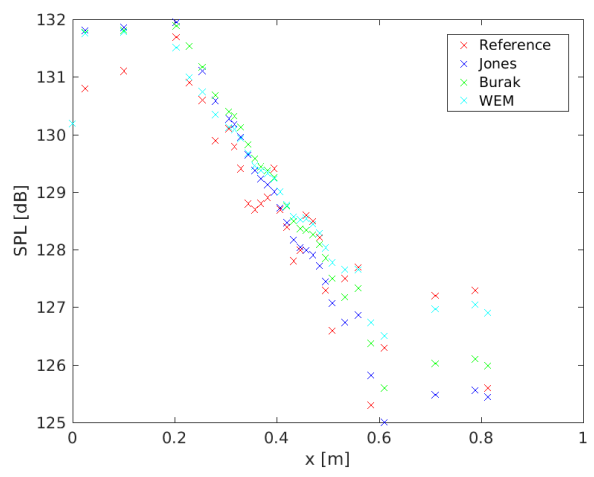

(d) $2000 \mathrm{~Hz}$

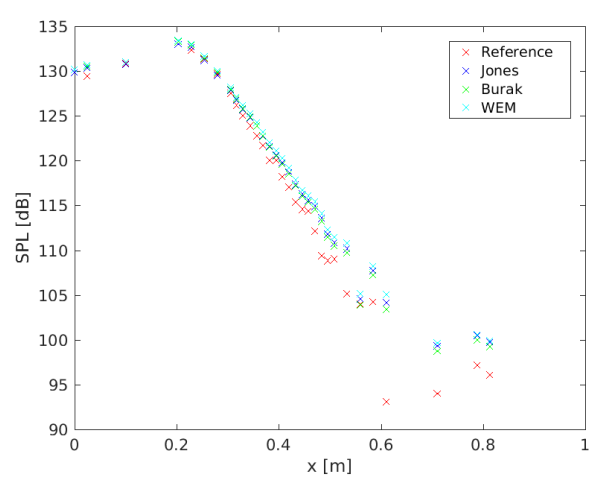

(f) $3000 \mathrm{~Hz}$

Figure 5.8: SPL for the impedance sensitivity study at $M=0.335$. 


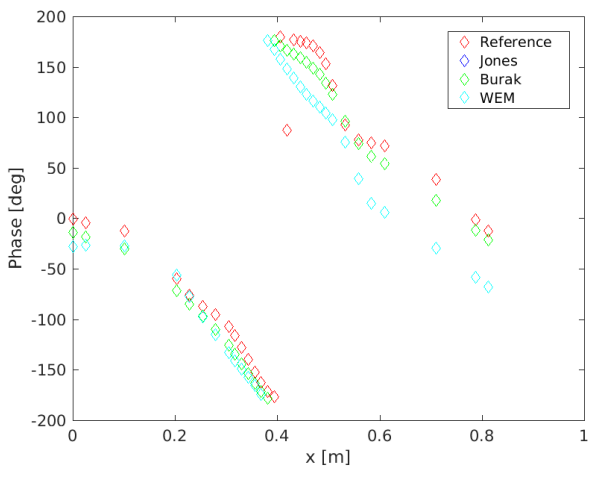

(a) $500 \mathrm{~Hz}$

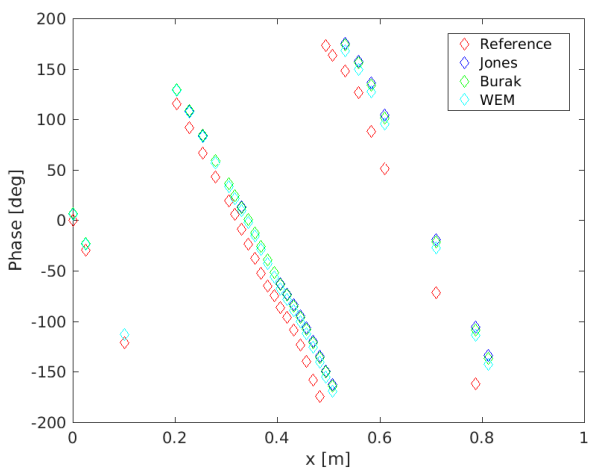

(c) $1500 \mathrm{~Hz}$

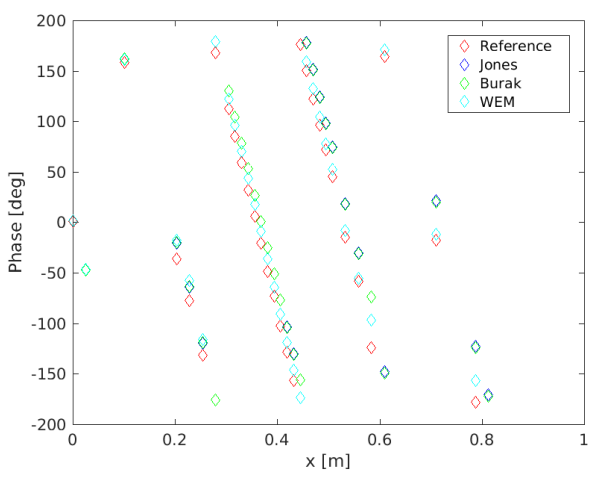

(e) $2500 \mathrm{~Hz}$

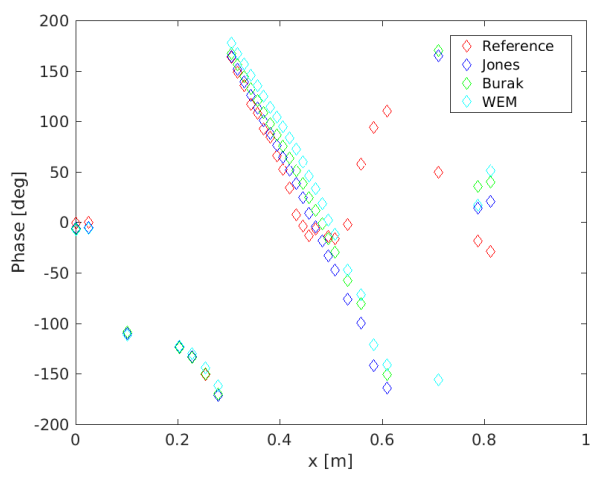

(b) $1000 \mathrm{~Hz}$

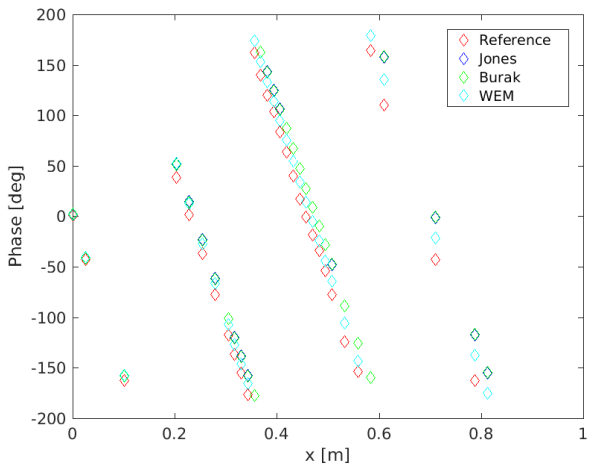

(d) $2000 \mathrm{~Hz}$

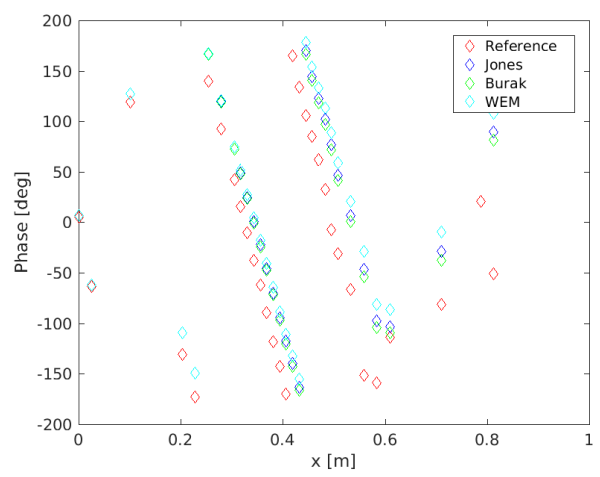

(f) $3000 \mathrm{~Hz}$

Figure 5.9: Phase for the impedance sensitivity study at $M=0.335$. 


\subsection{Study Case 1}

The main goal of this study is to determine the scattering matrix for all frequencies and to compare the obtained values with, both experimental and numerical data, provided by [30]. In that work two different values for the impedance are obtained. Despite being an unphysical condition, both values are taken into consideration. The motivation for this procedure is discussed in Chapter 6. However, for the sake of clarity, only the values educed with an upstream source are plotted. In order to have a better understanding of the results, the different components of the scattering matrix are plotted separately. Accordingly, the upstream and downstream transmission coefficients $\left(T^{+}, T^{-}\right)$are shown in Fig 5.10 and Fig 5.11. Likewise, the upstream and downstream reflection coefficients $\left(R^{+}, R^{-}\right)$are presented in Fig 5.12 and Fig 5.13. It is possible to see that the values obtained for $T^{+}$and $R^{-}$are close to the experimental values when a time domain is considered. The equivalent frequency domain solution offers values close to the experimental and the time domain ones. In the case of $T^{+}$the value corresponding to $2000 \mathrm{~Hz}$ is off. If $R^{-}$is considered, the same occurs at $500 \mathrm{~Hz}$ and $2500 \mathrm{~Hz}$. Nonetheless, this agreement is not as good for $T^{-}$and $R^{+}$where a time domain is considered since large discrepancies with respect to the experimental values are found. In this two cases, the frequency domain solution performs better and shows an improvement with respect the time domain solution.

\subsection{Study Case 2}

In Fig 5.14 and Fig 5.15 the SPL and phase for a Mach number of 0.172 are shown respectively. On the other hand, Fig 5.16 and Fig 5.17 present the SPL and phase for a Mach number of 0.335 separately. A clear contrast is seen between both cases, being more obvious the differences in the compressible case. Nonetheless, a more thorough discussion is given in Chapter 6 . 


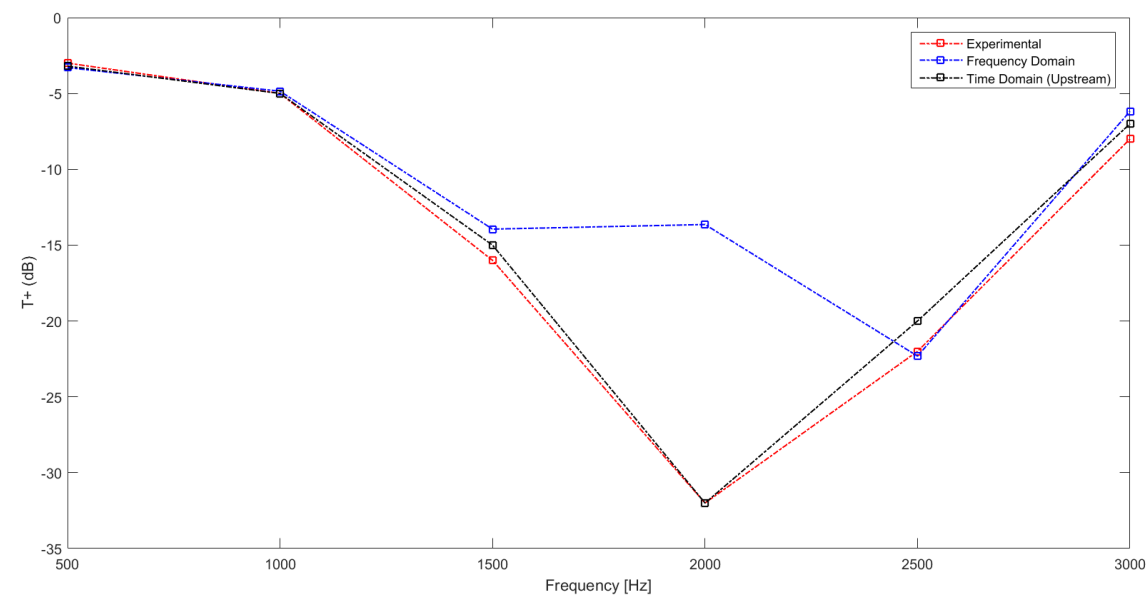

Figure 5.10: Transmission coefficient for an upstream source considering a) experimental data (in red); b) Frequency domain data (in blue); c) Time domain data (in black)

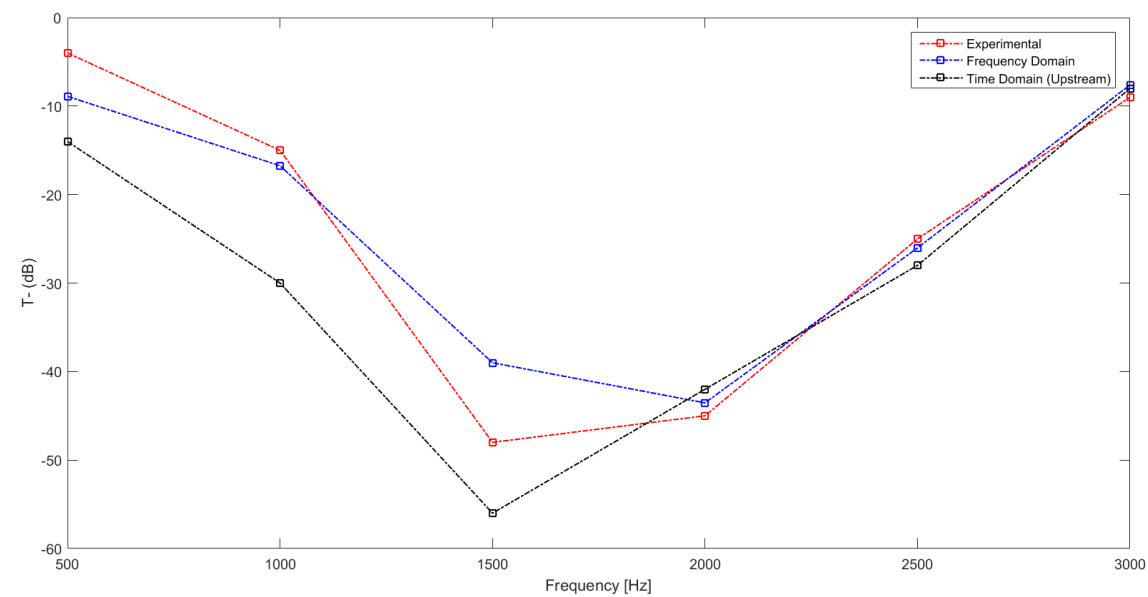

Figure 5.11: Transmission coefficient for a downstream source considering a) experimental data (in red); b) Frequency domain data (in blue); c) Time domain data (in black) 


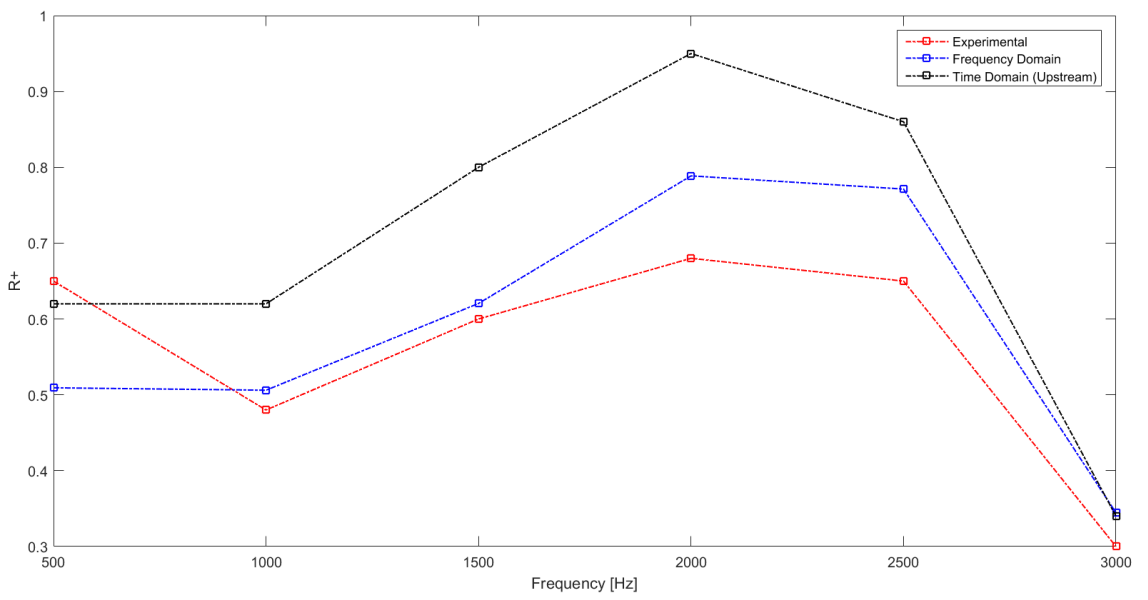

Figure 5.12: Reflection coefficient for an upstream source considering a) experimental data (in red); b) Frequency domain data (in blue); c) Time domain data (in black)

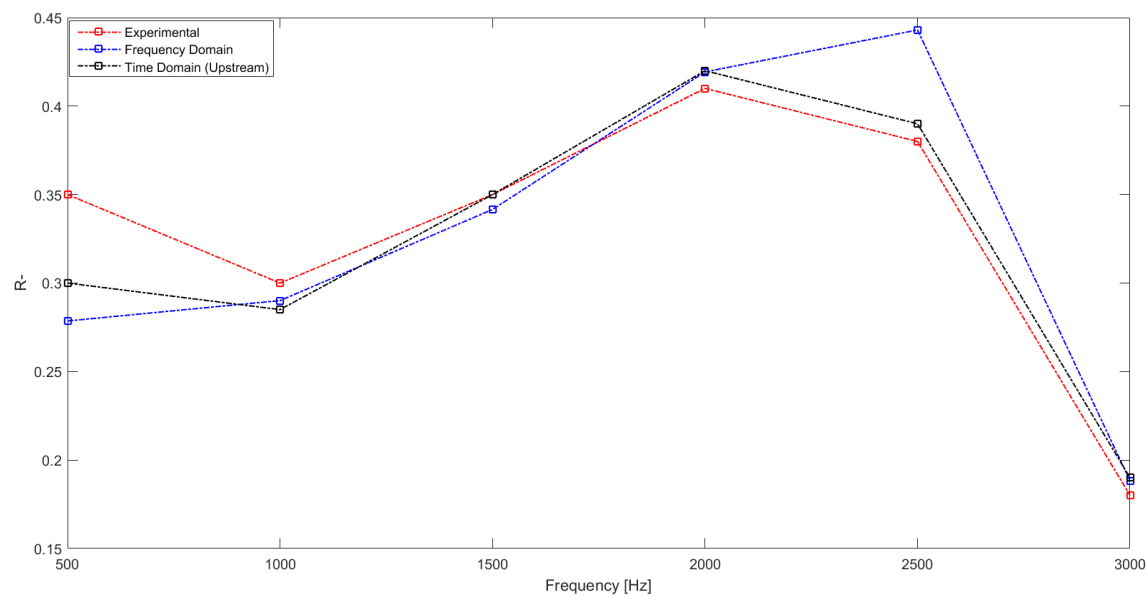

Figure 5.13: Reflection coefficient for a downstream source considering a) experimental data (in red); b) Frequency domain data (in blue); c) Time domain data (in black) 


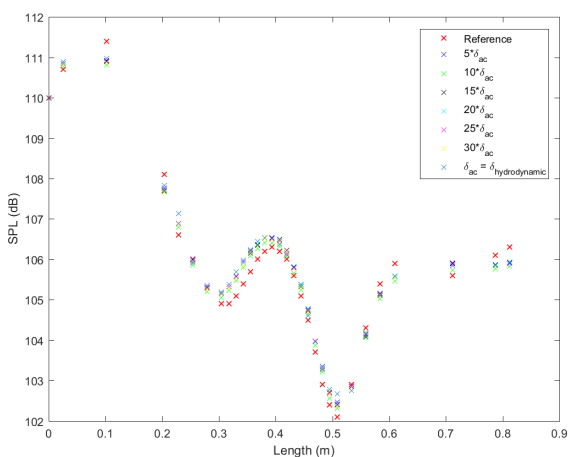

(a) $500 \mathrm{~Hz}$

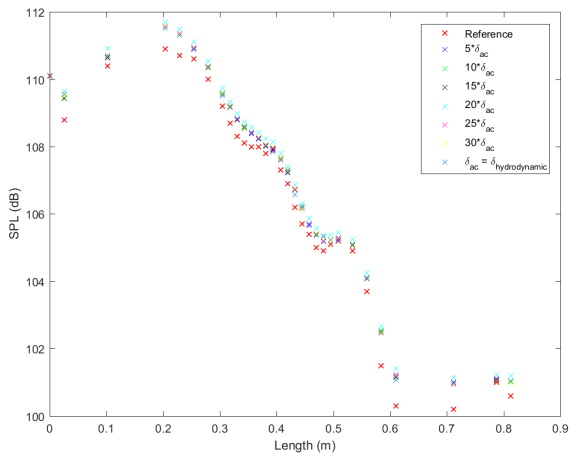

(c) $1500 \mathrm{~Hz}$

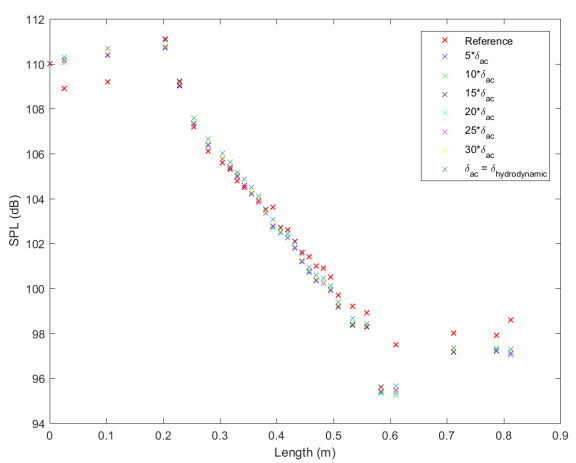

(e) $2500 \mathrm{~Hz}$

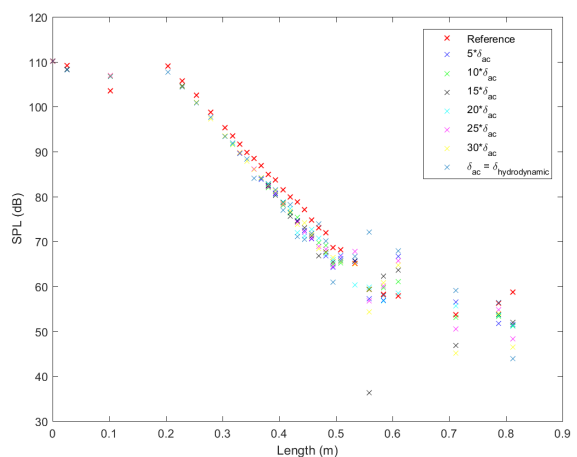

(b) $1000 \mathrm{~Hz}$

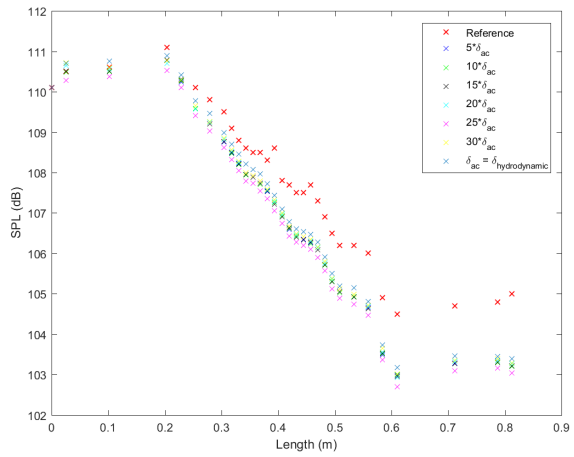

(d) $2000 \mathrm{~Hz}$

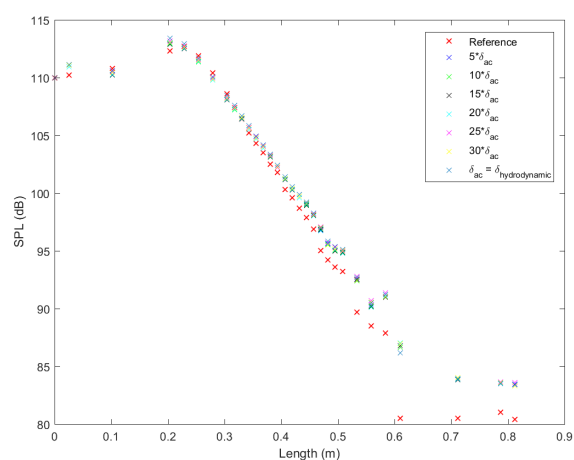

(f) $3000 \mathrm{~Hz}$

Figure 5.14: SPL for the study case 2 at $M=0.172$. 


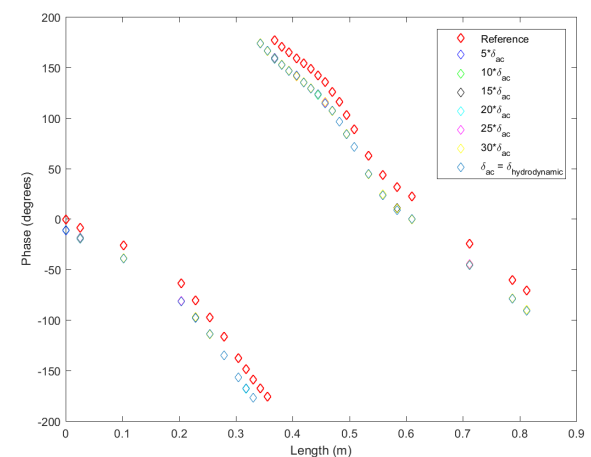

(a) $500 \mathrm{~Hz}$

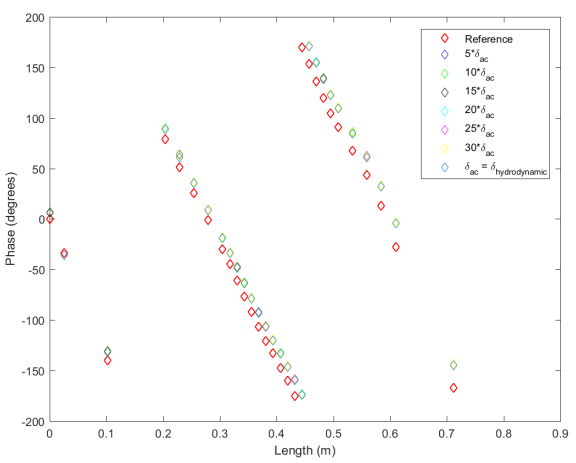

(c) $1500 \mathrm{~Hz}$

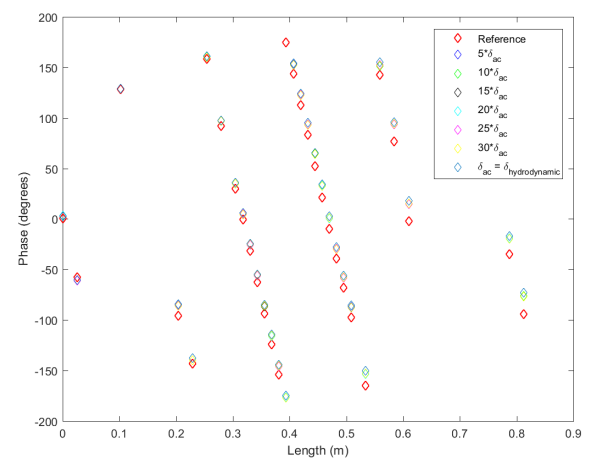

(e) $2500 \mathrm{~Hz}$

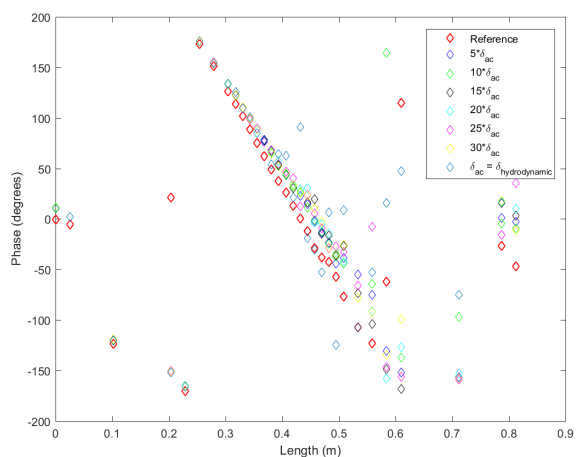

(b) $1000 \mathrm{~Hz}$

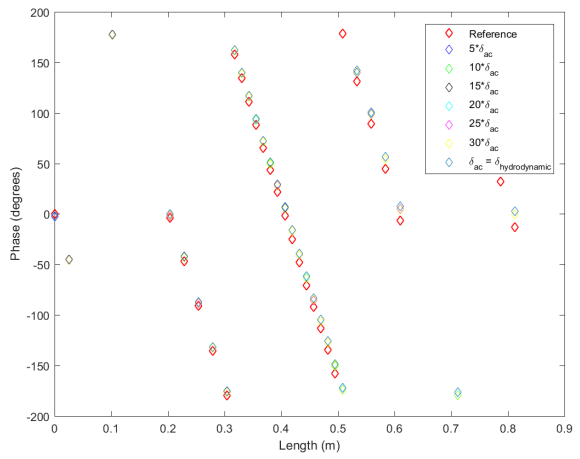

(d) $2000 \mathrm{~Hz}$

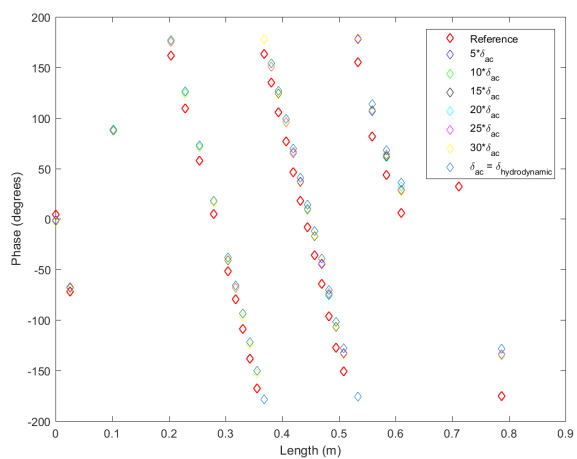

(f) $3000 \mathrm{~Hz}$

Figure 5.15: Phase for the study case 2 at $M=0.172$. 


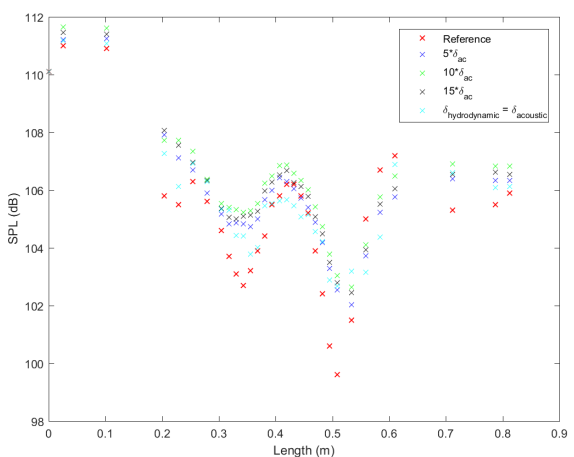

(a) $500 \mathrm{~Hz}$

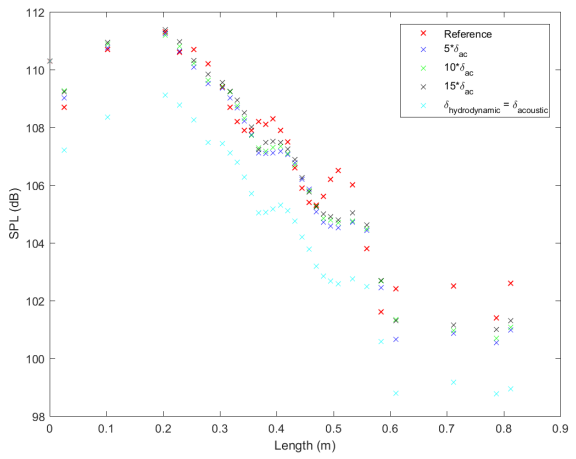

(c) $1500 \mathrm{~Hz}$

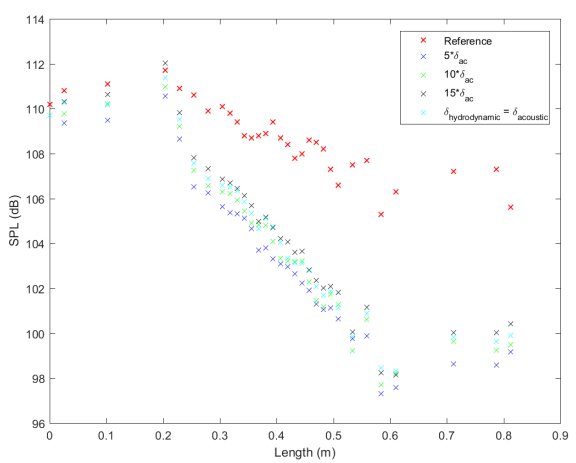

(e) $2500 \mathrm{~Hz}$

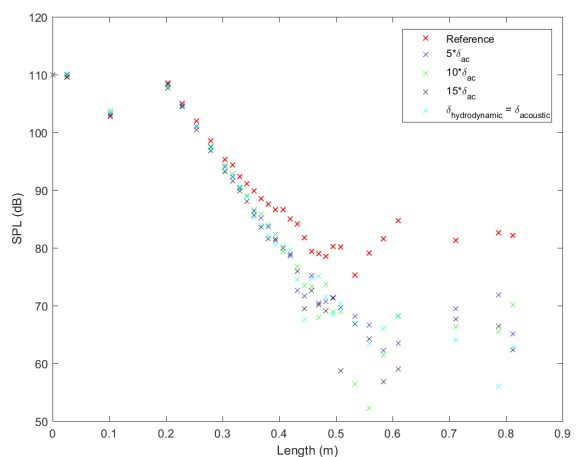

(b) $1000 \mathrm{~Hz}$

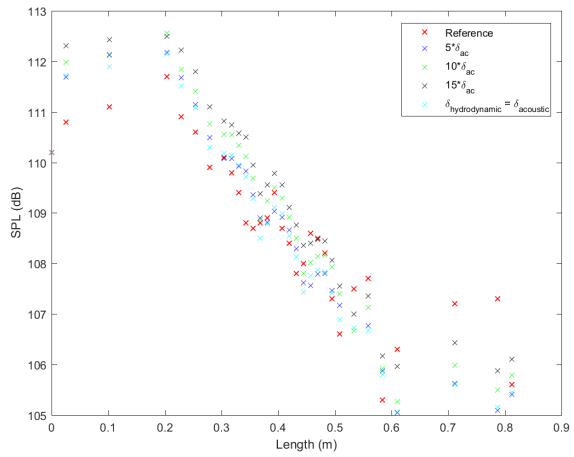

(d) $2000 \mathrm{~Hz}$

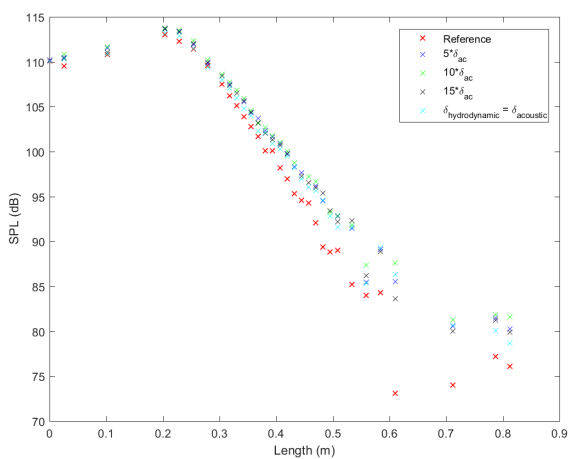

(f) $3000 \mathrm{~Hz}$

Figure 5.16: SPL for the study case 2 at $M=0.335$. 


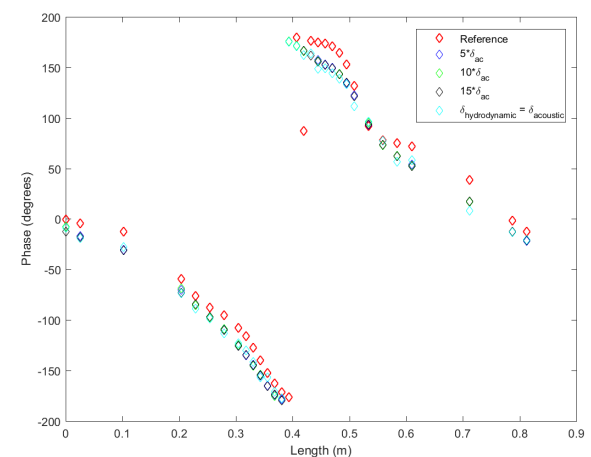

(a) $500 \mathrm{~Hz}$

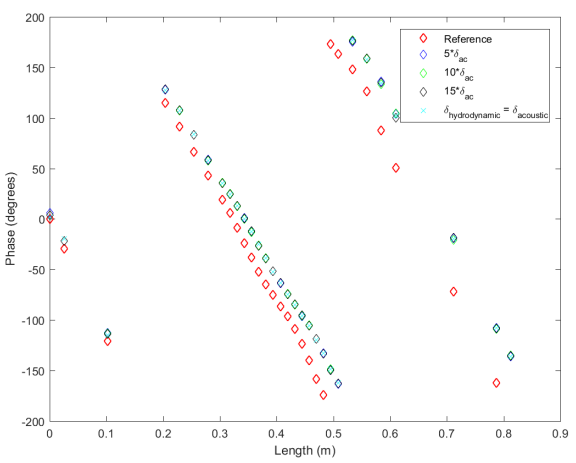

(c) $1500 \mathrm{~Hz}$

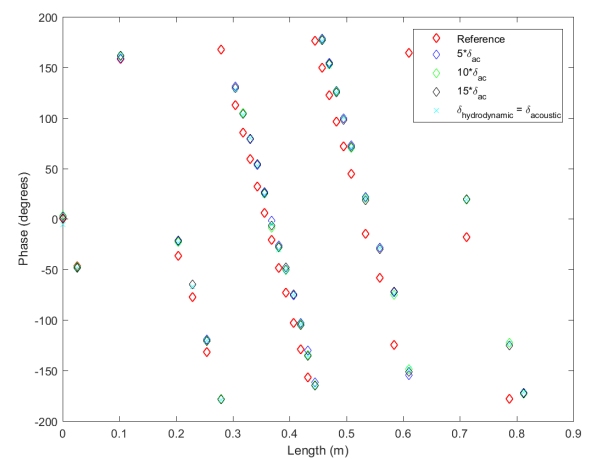

(e) $2500 \mathrm{~Hz}$

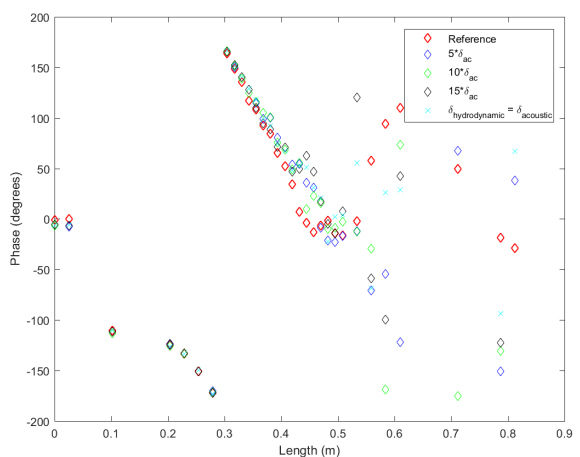

(b) $1000 \mathrm{~Hz}$

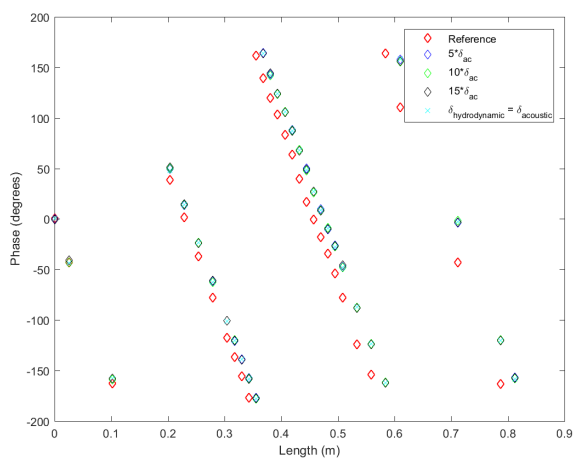

(d) $2000 \mathrm{~Hz}$

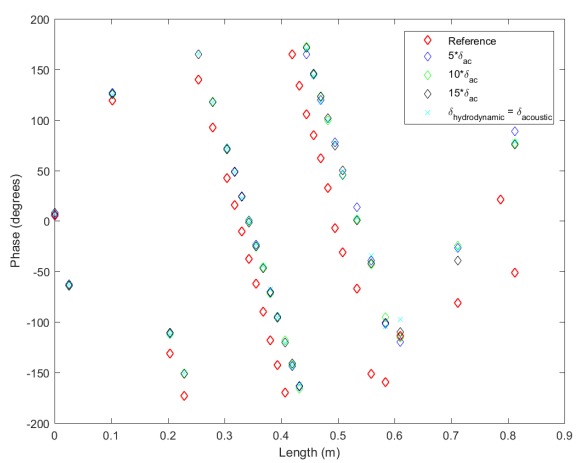

(f) $3000 \mathrm{~Hz}$

Figure 5.17: Phase for the study case 2 at $M=0.335$. 


\section{Chapter 6}

\section{Discussion}

Two test cases have been run in order to create a model capable of obtaining accurate and reliable results. In the first test case no flow through the duct is considered, i.e. the Mach number is 0. In Fig 5.2 and Fig 5.3 the SPL and the phase are plotted respectively. It can be seen that there is a very close agreement with the experimental data. Deviations are very low with a maximum of $2.05 \mathrm{~dB}$ for a frequency of $1000 \mathrm{~Hz}$ at the exit of the liner. The rest of frequencies show deviations lower than $1 \mathrm{~dB}$. Moreover, the model follows a trend similar to the experimental one at every frequency, and thus, it can be ascertain that the impedance value is working properly and that the model is fully capturing the behaviour at the locally reacting wall when there is no presence of a background flow.

The solution obtained in the second test case presents a good agreement with the experimental data as well. In Fig 5.4 and Fig 5.5 it is possible to appreciate that the simulation follows the trend established by the lined wall in the test section for every frequency studied. In addition, deviations at the exit of the liner are not too high from the experimental values with a maximum value of $3.64 \mathrm{~dB}$ for a frequency of $3000 \mathrm{~Hz}$. It is worth to mention that this behaviour is observed also in other studies found in the literature. Besides, since the boundary layer is well resolved the no-flow impedance was used for all frequencies instead of the impedance educed at the corresponding Mach number. The good agreement found with the experimental results validates this approach. Additionally, in Appendices A, B and C the graphs corresponding to Mach numbers $0.079,0.172$ and 0.255 respectively show the same trends already discussed. An impedance sensitivity study was done in order to validate the model. Different impedance values are evaluated and the results, presented in Fig (5.6 - 5.9), indicate that the model is predicting results similar to the ones found in the literature. Nonetheless, it is observed that all frequencies give good results except for $1000 \mathrm{~Hz}$ where discrepancies are clearly noticeable. In the literature it is found that different experimental studies report a hydrodynamic instability occurring at this frequency. However, since a frequency domain is used the instability is not present. As a consequence, the obtained solution is far from 
the experimental observations, making it complicated to obtain good and accurate results. Despite the latter it can be ascertained that both, the impedance value and the model, perform well capturing the viscous acoustics at the lined wall when a background flow is considered.

The purpose of the first study case was to check whether a viscous solution solution in the frequency domain could provide an improvement with respect a more general approach, consisting of an inviscid domain solution in the time domain, with the aid of the Myers impedance boundary condition to account for the effects created by the viscous boundary layer. In [30] two different values for the impedance boundary condition are provided. This unphysical result proved that the Myers boundary condition was not providing a good approximation of the near wall effects. Those impedance values where used to calculate the scattering matrix, and no good agreement with the experimental data was found. Hence, to prove the validity of this new approach, the same procedure was implemented. The two impedance values, upstream and downstream educed, were used to calculate the scattering matrix. The different components of the scattering matrix were plotted in Fig (5.10 - 5.13). Time domain values were in close agreement for $T^{+}$and $R^{-}$and the corresponding frequency domain show also good agreement in general. A significant improvement was found with the values obtained for $T^{-}$and $R^{+}$obtaining values that are close to the experimental ones. Regarding the downstream educed impedance, results do not indicate a good agreement either with the experimental data or the time domain approach for low frequencies. Nonetheless, at high frequencies the same values, as the upstream educed case, are obtained since both impedance values are the same. This means that for a unique impedance value the same scattering matrix is obtained in both directions which is logical since the problem is symmetric, i.e the sound propagation should not be affected by the source location. Therefore, the assumption of a viscous solution demonstrate to offer better results in general than the equivalent time domain simulation for the upstream educed values Another important conclusion from this study is that despite the Myers impedance boundary condition is ill-posed, and therefore not suitable to be used in impedance eduction methods, the impedance values obtained can still be used and provide better results if a viscous solution is adopted instead of the more general inviscid approach.

A second study case was done in order to assess the importance of the acoustic boundary layer in the calculations. Two cases, with Mach number 0.172 and 0.335 respectively, were considered. The acoustics are solved in both cases using the compressible version of the linearised Navier-Stokes equations. Hence, the main difference between them is based on whether a compressible or incompressible background flow is adopted. Results are presented in Fig (5.14 - 5.17). For the incompressible case no big discrepancies are observed and the obtained values are very close to the experimental ones. Only at $2000 \mathrm{~Hz}$ it is found that the values do not follow the trend properly and deviations are a bit higher with respect the experimental and the obtained solution $(3 \mathrm{~dB})$. For the compressible case differences 
are clearly noticeable although the variation with respect to the solution obtained with the acoustic boundary layer corresponding to $3000 \mathrm{~Hz}$ is not high. The biggest difference is observed in Fig 5.16e although the deviation with respect the experimental value at the exit of the liner is $2 \mathrm{~dB}$. This values is higher than the value observed when the most critical first layer thickness is considered but is still acceptable. In addition, despite only the acoustic boundary layer is modified in the whole study, the assumption of compressible or incompressible background flow may have an effect on the solutions and this may be why certain frequencies experience the variation on one configuration and nothing happens in the other. However, in general it can be ascertained that the effect of not considering the boundary layer is not that crucial to achieve good results and the general assumptions that are taken in most of the works found in the literature are justified since as long as the right impedance value is used good and accurate results should be expected. 



\section{Chapter 7}

\section{Conclusions}

A computational study was performed using Comsol Multiphysics to study the near wall effects at the local reacting wall of an acoustic liner. The Linearised NavierStokes equations were chosen to study the sound propagation since no assumptions are made for the boundary layer which, in turn, is fully resolved for both the hydrodynamic and acoustic fields. Two test cases were run in order to validate a model. The comparison with the experimental values presented by [14] showed a good agreement and proved that the viscous acoustics were captured properly either with or without background flow. Problems arouse when considering a frequency of $1000 \mathrm{~Hz}$ since the usage of a frequency-domain does not allow instabilities to grow. Both SPL and phase plots shown that there is not a good agreement with the experimental data. This conclusion was reached as well by other researchers that also reported problems when analysing the sound dissipation for this frequency. In this case, the reason is that the natural hydrodynamic instability occurring at that frequency is not captured in the simulations since the usage of a frequency domain does not allow instabilities to grow, therefore, making it complicated to obtain results close to the experimental ones. In the first test case, simulations were done to determine whether a viscous approach could render better results than the equivalent inviscid simulations in time domain. The study shows that an improvement, with respect the inviscid time domain simulations, is achieved when the upstream educed impedance values are used. For the downstream ones no good agreement is found at low frequencies, whereas at high frequencies the same values for the scattering matrix are obtained. This is an expected outcome since it is unphysical to have two different values for the impedance. Hence, despite the Myers impedance value is illposed and proved not to describe the near wall effects properly, it can still be used in numerical impedance eduction codes and the obtained values render good results if a viscous solution is adopted. Further work could be done educing impedances considering viscosity. A second test case was run to determine the importance of the acoustical boundary layer in the simulations. Results show that it is not a crucial factor in order to achieve a good solution. In turn, as long as the impedance value is properly determined, the right number of elements per wavelength and scheme 
order are used, an accurate outcome should be expected. The frequency domain is shown to be able to offer correct solutions with a low computational effort hence being a good optimisation tool for modelling intake liners in aircraft engines. 
Appendix A

Data for Test Case 2 with $M=0.079$ 


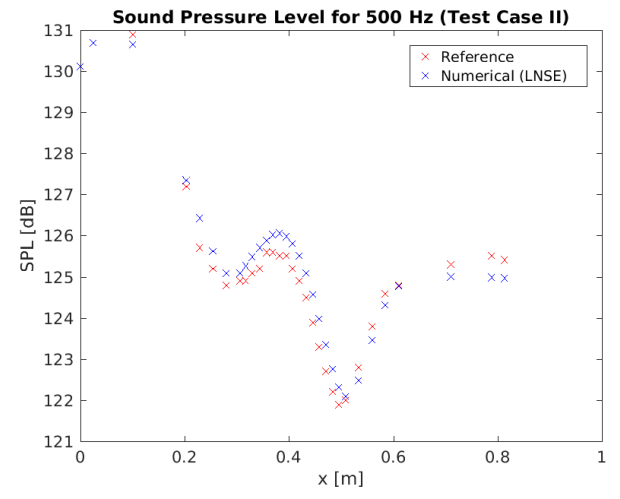

(a) $500 \mathrm{~Hz}$

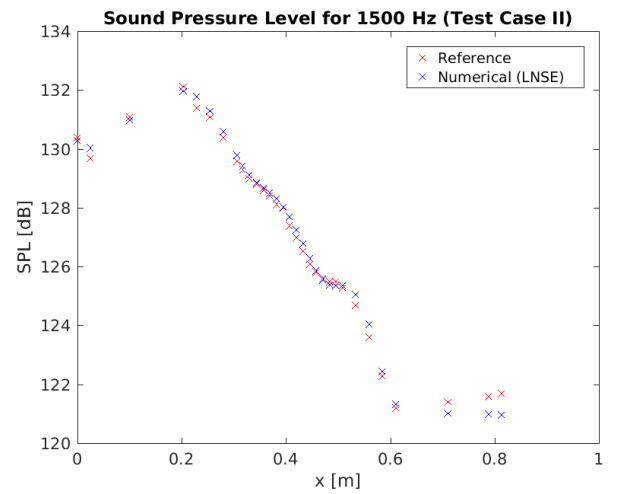

(c) $1500 \mathrm{~Hz}$

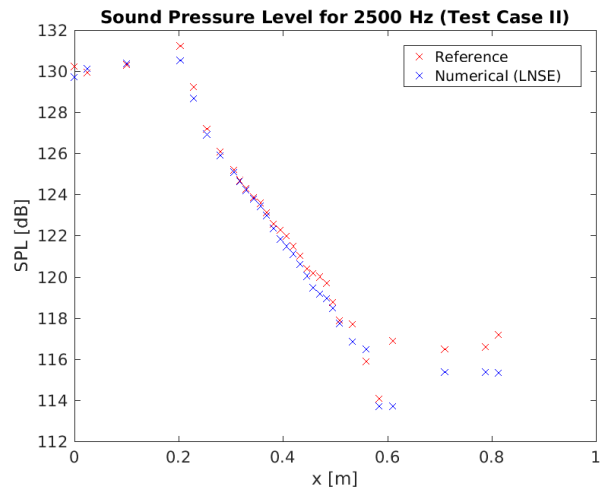

(e) $2500 \mathrm{~Hz}$

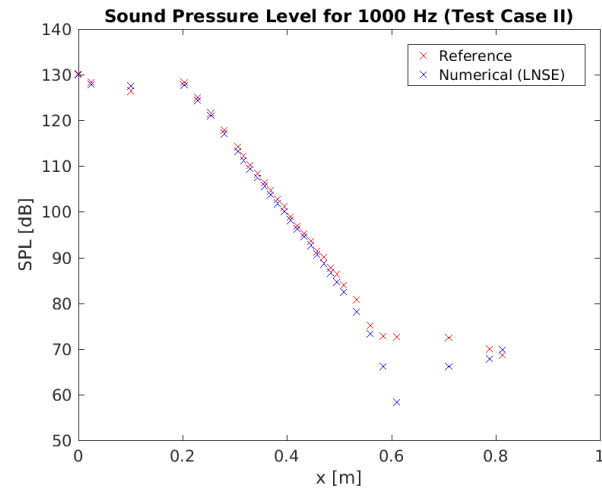

(b) $1000 \mathrm{~Hz}$

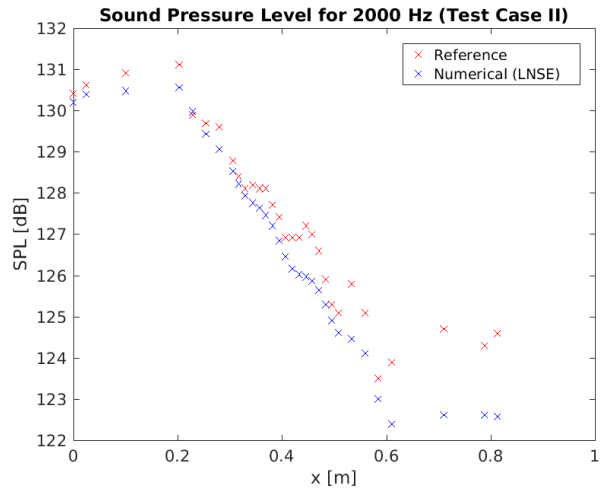

(d) $2000 \mathrm{~Hz}$

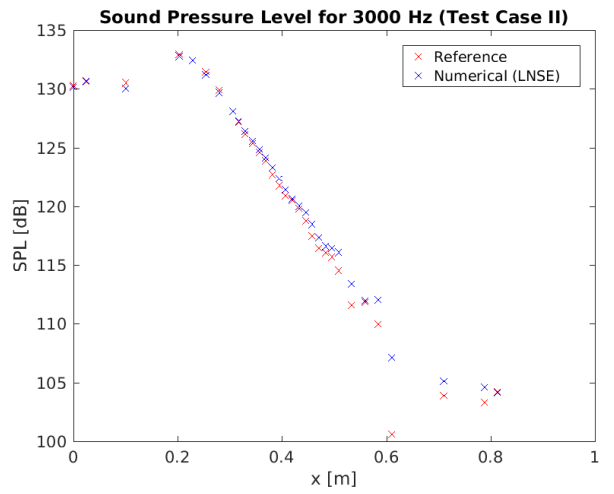

(f) $3000 \mathrm{~Hz}$

Figure A.1: Measured Sound Pressure Level in Test Case 2 with $\mathrm{M}=0.079$ 


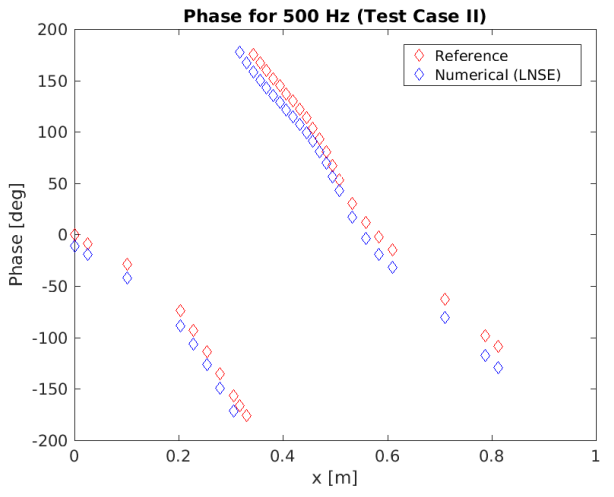

(a) $500 \mathrm{~Hz}$

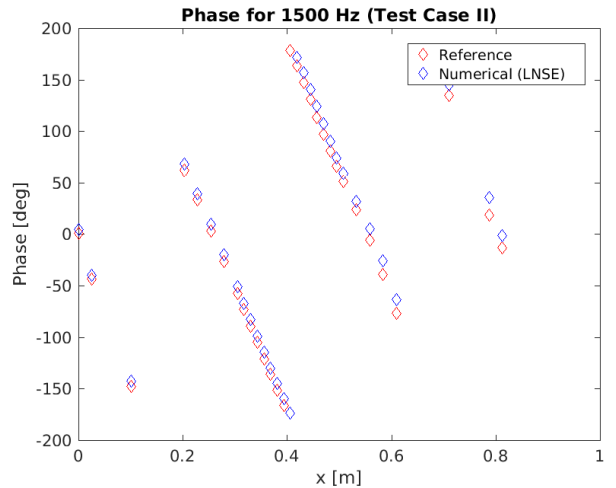

(c) $1500 \mathrm{~Hz}$

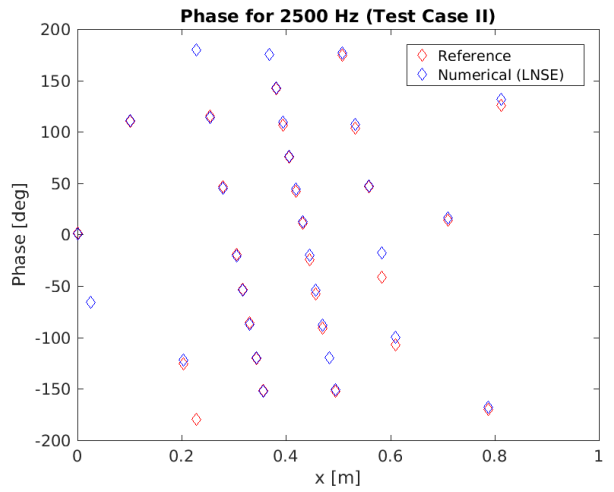

(e) $2500 \mathrm{~Hz}$

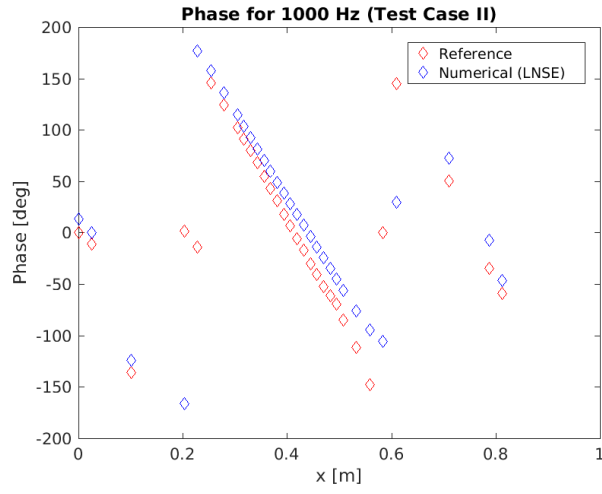

(b) $1000 \mathrm{~Hz}$

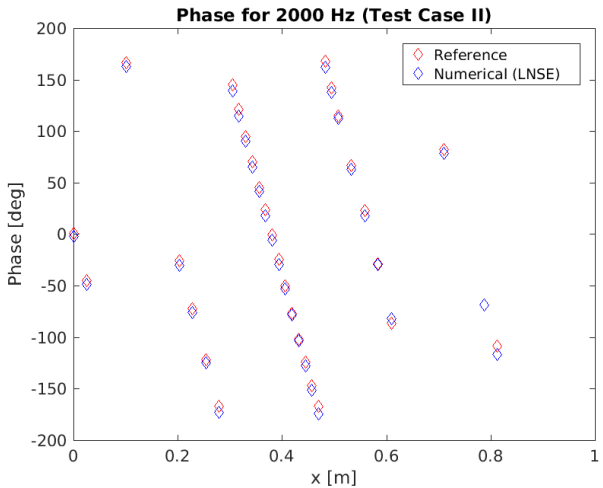

(d) $2000 \mathrm{~Hz}$

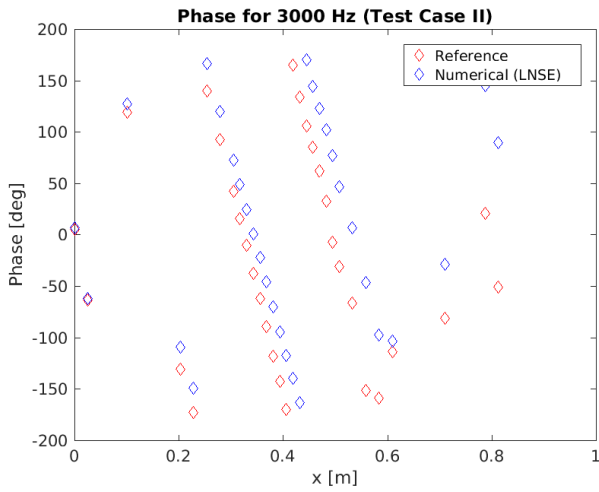

(f) $3000 \mathrm{~Hz}$

Figure A.2: Measured Phase in Test Case 2 with $\mathrm{M}=0.079$ 



\section{Appendix B}

Data for Test Case 2 with $\mathrm{M}=0.172$ 


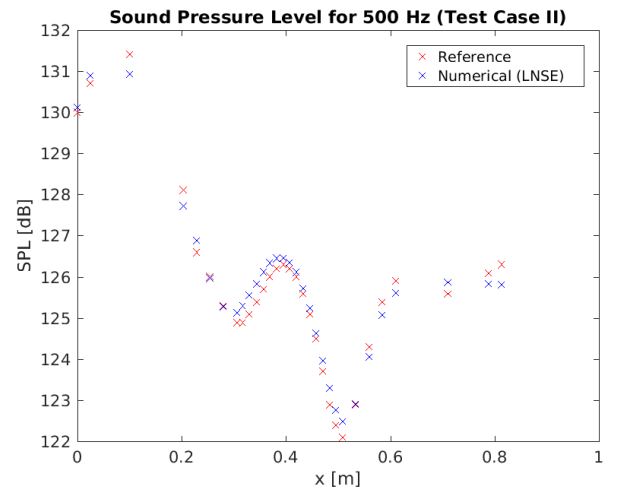

(a) $500 \mathrm{~Hz}$

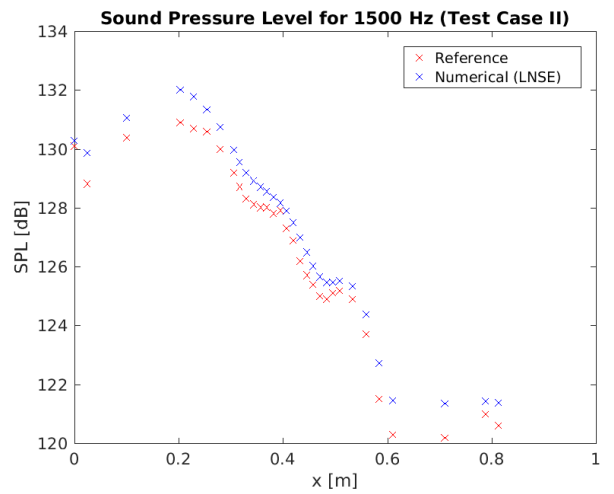

(c) $1500 \mathrm{~Hz}$

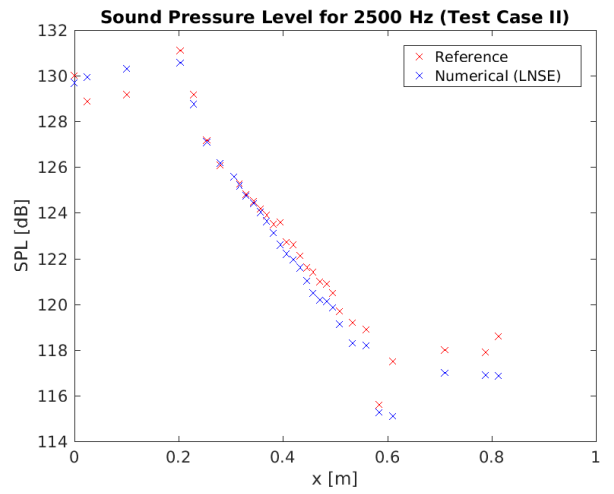

(e) $2500 \mathrm{~Hz}$

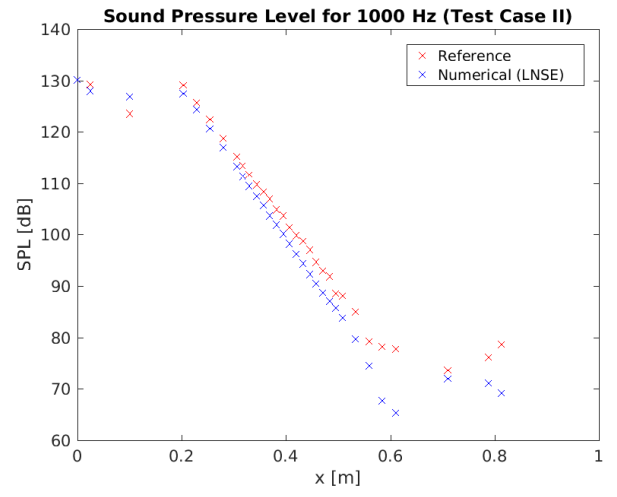

(b) $1000 \mathrm{~Hz}$

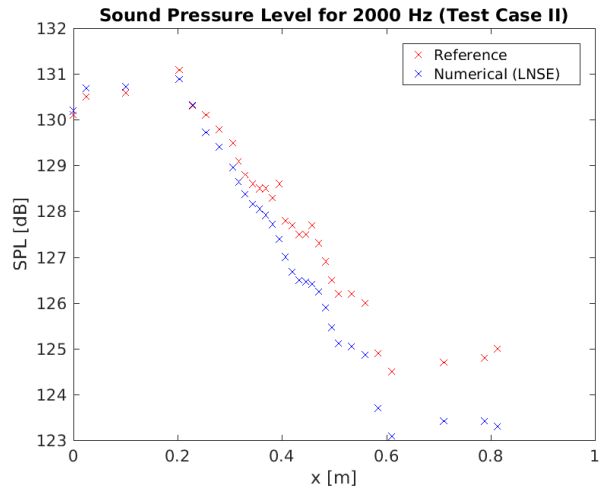

(d) $2000 \mathrm{~Hz}$

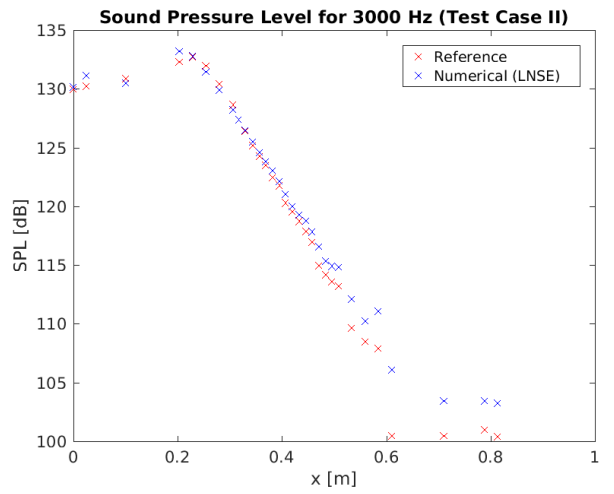

(f) $3000 \mathrm{~Hz}$

Figure B.1: Measured Sound Pressure Level in Test Case 2 with $\mathrm{M}=0.172$ 


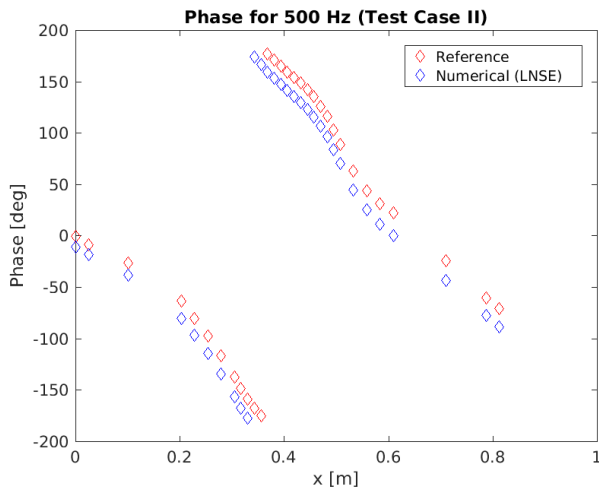

(a) $500 \mathrm{~Hz}$

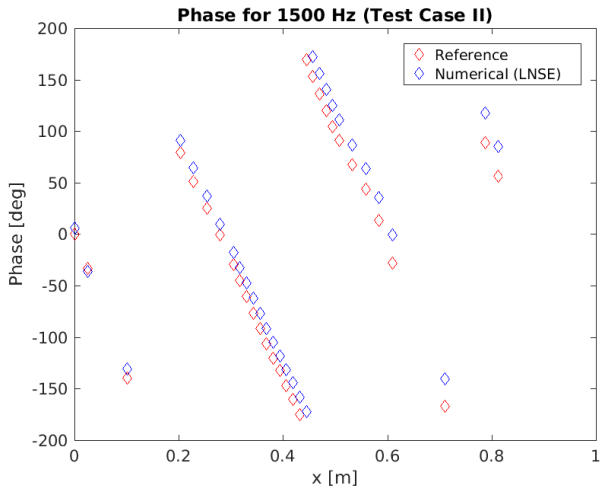

(c) $1500 \mathrm{~Hz}$

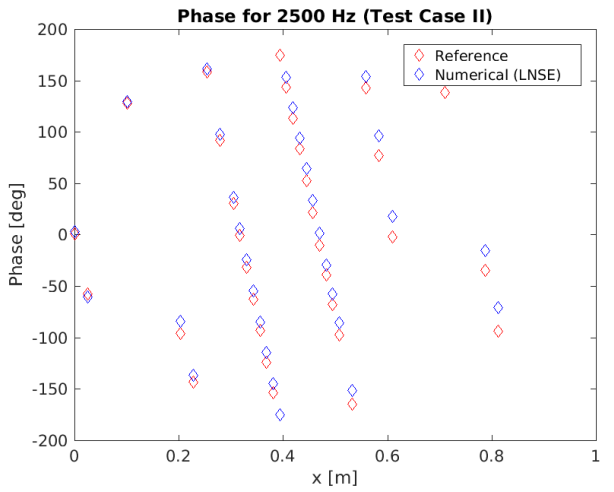

(e) $2500 \mathrm{~Hz}$

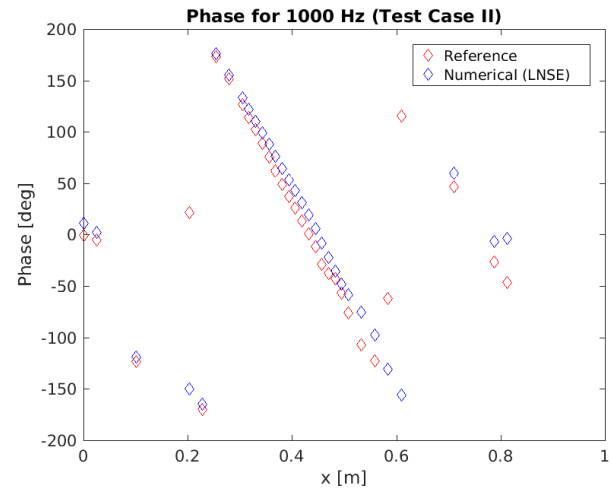

(b) $1000 \mathrm{~Hz}$

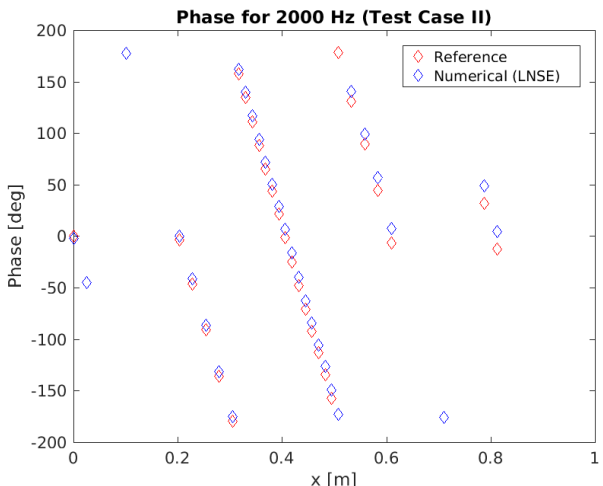

(d) $2000 \mathrm{~Hz}$

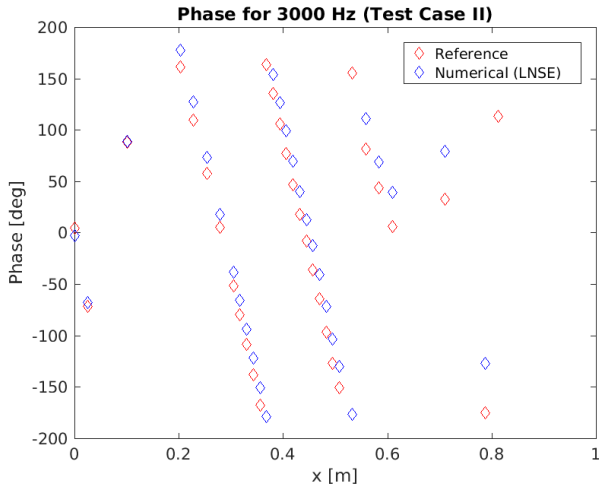

(f) $3000 \mathrm{~Hz}$

Figure B.2: Measured Phase in Test Case 2 with $\mathrm{M}=0.172$ 

Appendix C

Data for Test Case 2 with $M=0.255$ 


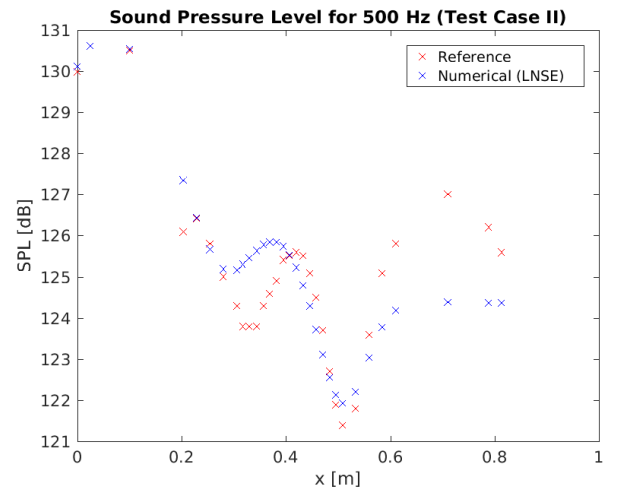

(a) $500 \mathrm{~Hz}$

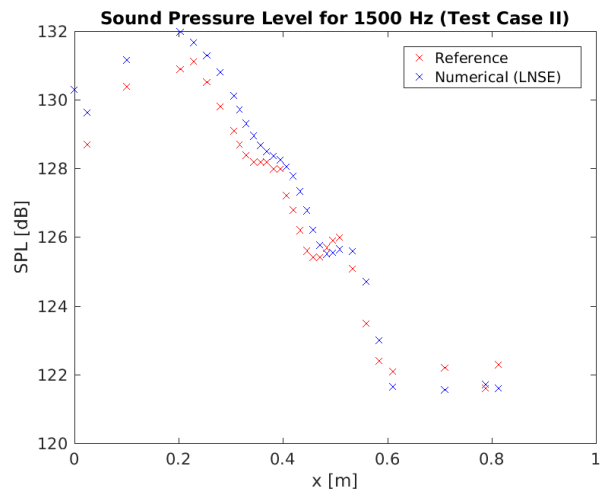

(c) $1500 \mathrm{~Hz}$

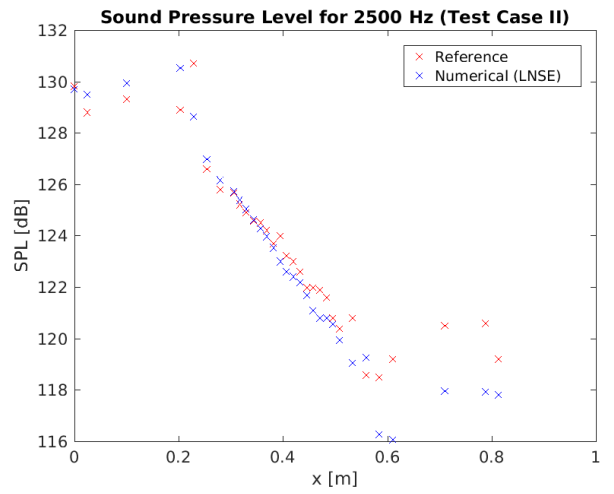

(e) $2500 \mathrm{~Hz}$

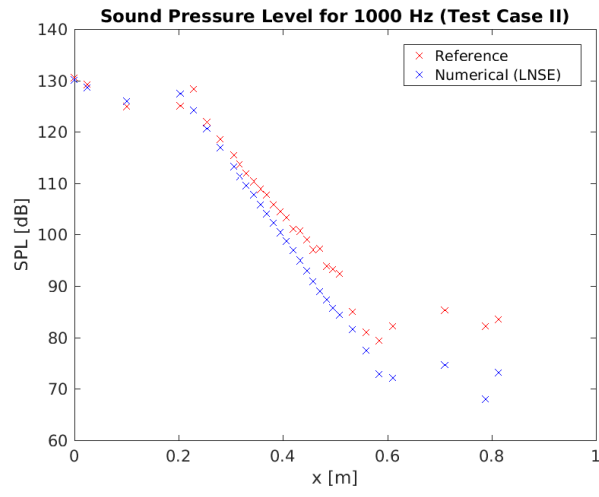

(b) $1000 \mathrm{~Hz}$

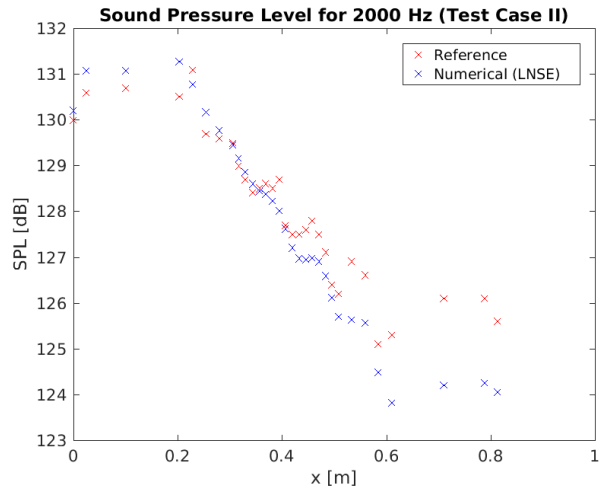

(d) $2000 \mathrm{~Hz}$

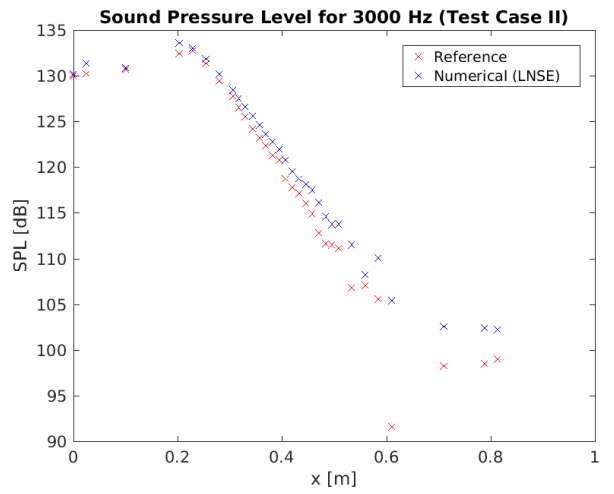

(f) $3000 \mathrm{~Hz}$

Figure C.1: Measured Sound Pressure Level in Test Case 2 with $\mathrm{M}=0.255$ 


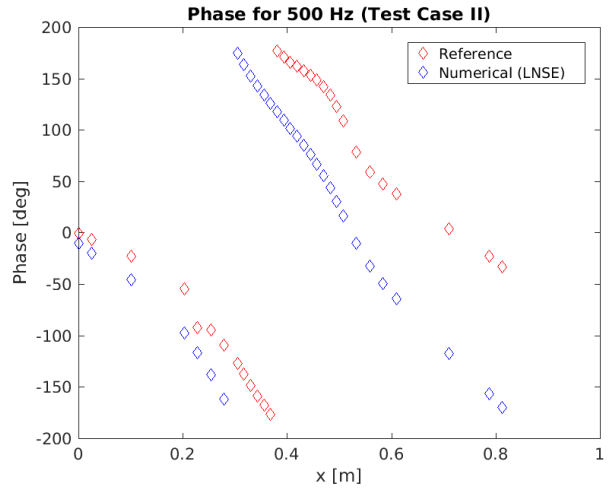

(a) $500 \mathrm{~Hz}$

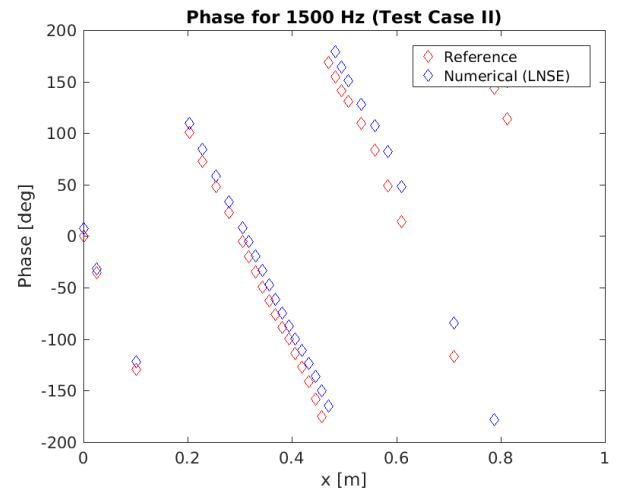

(c) $1500 \mathrm{~Hz}$

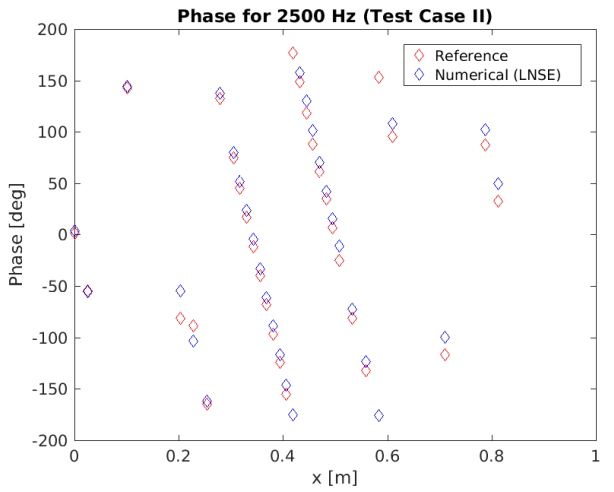

(e) $2500 \mathrm{~Hz}$

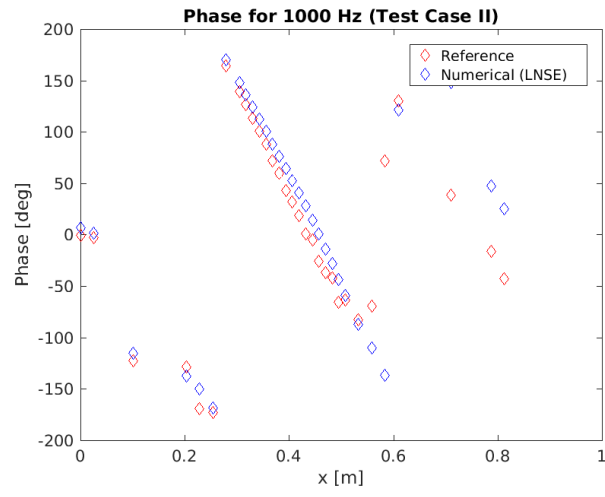

(b) $1000 \mathrm{~Hz}$

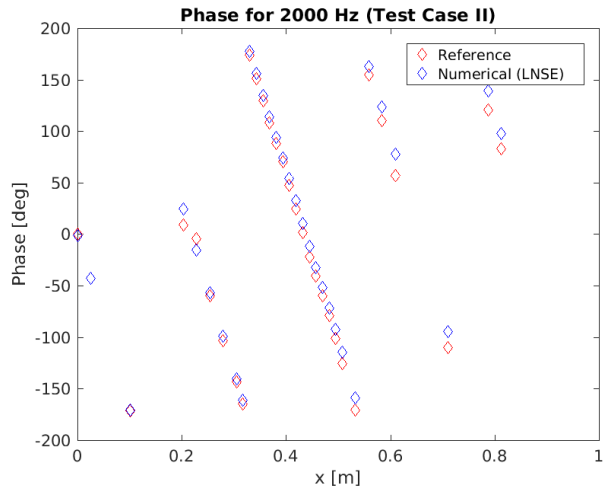

(d) $2000 \mathrm{~Hz}$

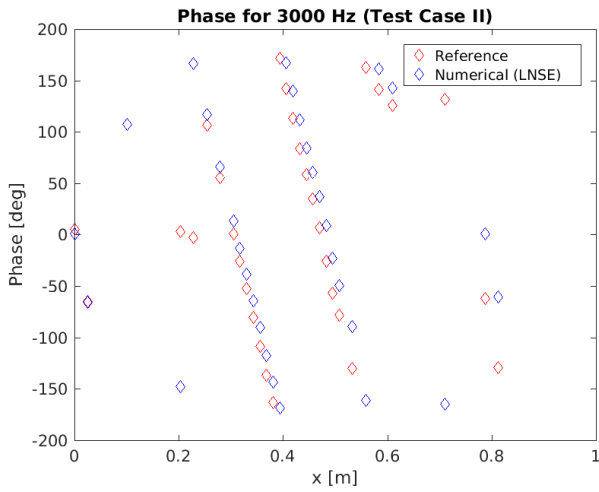

(f) $3000 \mathrm{~Hz}$

Figure C.2: Measured Phase in Test Case 2 with $\mathrm{M}=0.255$ 



\section{Bibliography}

[1] Mats Åbom. An introduction to Flow Acoustics.

[2] Gunilla Efraimsson Axel Kierkegaard, Susann Boij. A frequency domain linearized navier-stokes equations approach to acoustic propagation in flow ducts with sharp edges. pages $710-719$.

[3] E. J. Brambley. Acoustic implications of a thin viscous boundary layer over a compliant surface of permeable liner. 678:348 - 378 .

[4] E. J. Brambley. Review of acoustic liner models with flow.

[5] E. J. Brambley. A well-posed boundary condition for acoustic liners in straigth ducts with flow. 49:1272 - 1282 .

[6] E. J. Brambley Doran Khamis. The effective impedance of a finite-thickness viscothermal boundary layer over an acoustic lining. 796:386 - 416 .

[7] T. Elnady. Modelling and characterization of perforates in lined ducts and mufflers.

[8] World Health Organization Regional Office for Europe. Noise: Data and statistics. URL http://www.euro.who.int/en/health-topics/ environment-and-health/noise/data-and-statistics.

[9] X. Zhang G. Ashcroft. Optimized prefactored compact schemes. 190:459 - 477.

[10] E. J. Brambley G. Gabard. A full disperions analysis of time-domain simulations of acoustic liners with flow. 273:310 - 326.

[11] Gwénaël Gabard. A comparison of impedance boundary conditions for flow acoustics. 332:714 - 724 .

[12] M. E. Goldstein. Aeroacoustics.

[13] W. Malalasekera H.K. Versteeg. An introduction to computational fluid dynamics. The finite volume method. Pearson Prentice Hall, 2nd edition.

[14] T. L. Parrott M. G. Jones, W. R. Watson. Benchmark data for evaluation of aeroacoustic propagation codes with grazing flow. 
[15] Lars-Erik Eriksson Stéphane Baralon Markus O. Burak, Mattias Billson. Validation of a time- and frequency-domain grazing flow acoustic liner model. 47 (8), August.

[16] B.M. Howerton-S. Busse-Gerstengarbe M.G. Jones, W.R. Watson. Comparative study of impedance eduction methods, part 2: Nasa tests and methodology. pages 2013-2125.

[17] M. K. Myers. On the acoustic boundary condition in the presence of flow. 71: $429-434$.

[18] Library of the European Parliament. New rules on eu airport noise restrictions.

[19] D. R. Dowling P. K. Kundu, I. M. Cohen. Fluid Mechanics. 5th edition.

[20] A. D. Pierce. Acoustics: An Introduction to Its Physical Principles and Applications.

[21] S. B. Pope. Turbulent Flows. 11th edition.

[22] Ciarán J. O'Reilly. A wave expansion method for acoustic propagation in lined flow ducts. 90:54-63.

[23] Christoph Richter. Liner impedance model in the time domain with flow.

[24] Christoph Richter. A review of time domain impedance boundary conditions. pages $3421-3427$.

[25] P. Mustafi R.J. Astley, R. Sugimoto. Computational aero-acoustics for fan duct propagation and radiation. current status and application to turbofan liner optimisation. 330:3832 - 3845 .

[26] A. V. Johansson S. Wallin. Turbulence Lecture Notes.

[27] Berry Bernard. Sanchez Diana. The economic valuation of aircraft noise effects: a critical review of the state of the art.

[28] Hermann Schlichting. Boundary-Layer Theory. McGraw Hill, 7th edition.

[29] Christopher K. W. Tam. Time-Domain Impedance Boundary Condition.

[30] Yves Aurégan Ygaäl Renou. Failure of the ingard-myers boundary condition for a lined duct: An experimental investigation. Journal of the Acoustical Society of America, 130(1), July 2011.

[31] Vincent Pagneux Yves Auregan, Rudolf Starobinski. Influence of grazing flow and dissipation effects on the acoustic boundary conditions at a lined wall. 109. 
www.kth.se 